\title{
ARTICLE Epithelial RABGEF1 deficiency promotes intestinal inflammation by dysregulating intrinsic MYD88-dependent innate signaling
}

Sophie El Abbas ${ }^{1,2}$, Coraline Radermecker ${ }^{1,2}$, Qiang Bai ${ }^{1}$, Charline Beguin ${ }^{1}$, Joey Schyns ${ }^{1,2}$, Margot Meunier ${ }^{1,2}$, Dimitri Pirottin ${ }^{2,3}$, Christophe J. Desmet ${ }^{2}$, Marie-Alice Meuwis ${ }^{4,5}$, Tatiana Art $^{2}$, Edouard Louis ${ }^{4,5}$, See-Ying Tam ${ }^{6,7}$, Mindy Tsai ${ }^{6,7}$, Fabrice Bureau ${ }^{2,3,8}$, Stephen J. Galli, ${ }^{6,7,9}$ and Thomas Marichal ${ }^{1,2,8}$

Intestinal epithelial cells (IECS) contribute to the regulation of intestinal homeostasis and inflammation through their interactions with the environment and host immune responses. Yet our understanding of IEC-intrinsic regulatory pathways remains incomplete. Here, we identify the guanine nucleotide exchange factor RABGEF1 as a regulator of intestinal homeostasis and innate pathways dependent on IECs. Mice with IEC-specific Rabgef1 deletion (called Rabgef1 ${ }^{\text {IEC-KO }}$ mice) developed a delayed spontaneous colitis associated with the local upregulation of IEC chemokine expression. In mouse models of colitis based on Interleukin-10 deficiency or dextran sodium sulfate (DSS) exposure, we found that IEC-intrinsic RABGEF1 deficiency exacerbated development of intestinal pathology and dysregulated IEC innate pathways and chemokine expression. Mechanistically, we showed that RABGEF1 deficiency in mouse IECs in vitro was associated with an impairment of early endocytic events, an increased activation of the p38 mitogenactivated protein kinase (MAPK)-dependent pathway, and increased chemokine secretion. Moreover, we provided evidence that the development of spontaneous colitis was dependent on microbiota-derived signals and intrinsic MYD88-dependent pathways in vivo. Our study identifies mouse RABGEF1 as an important regulator of intestinal inflammation, MYD88-dependent IEC-intrinsic signaling, and chemokine production. This suggests that RABGEF1-dependent pathways represent interesting therapeutic targets for inflammatory conditions in the gut.

Mucosal Immunology (2020) 13:96-109; https://doi.org/10.1038/s41385-019-0211-z

\section{INTRODUCTION}

The intestinal mucosa is particularly exposed to microorganisms and foreign antigens. Intestinal homeostasis thus relies on finely tuned interactions between the environment, intestinal epithelial cells (IECs) and host immune responses, which allow the host to assimilate nutrients and to tolerate commensal microorganisms and dietary antigens, while mounting appropriate defense responses against pathogens. ${ }^{1-3}$

IECs critically contribute to the regulation of intestinal homeostasis and inflammation. ${ }^{2,3}$ Indeed, in addition to forming a physical barrier and eliciting efficient secretory defenses, IECs express a wide range of pattern recognition receptors (PRRs) ${ }^{2,4-6}$ that can sense microbiota-derived signals and integrate them into appropriate homeostatic or defensive responses. ${ }^{2,3,7}$ The abundance of symbiotic microorganisms necessitates that IECs express negative regulators of PRR signaling pathways in order to avoid aberrant immune responses and a break of tolerance, which can be associated with chronic inflammatory conditions such as inflammatory bowel diseases (IBDs). Experimental models of IBDs have substantially contributed to our understanding of IBD pathogenesis, which is thought to be driven by interactions between microbial, environmental, and host genetic factors. $2,5,8-12$

RAB guanine nucleotide exchange factor 1 (RABGEF1) was first identified as a GDP/GTP exchange factor for the small GTPase Rab5, which regulates endocytosis and early endosome trafficking events. ${ }^{13-17}$ RABGEF1 contains several functional domains, including a N-terminal A20-like zinc finger domain that can exhibit E3 ubiquitin ligase activity ${ }^{18,19}$ and a Vps9 domain promoting GEF activity for Rab5. ${ }^{13-15}$ RABGEF1 is critical for health, as globally RABGEF1-deficient mice exhibit perinatal mortality. ${ }^{20}$ In addition, we showed that RABGEF1 expression within skin epithelial cells (i.e., keratinocytes) is required to sustain optimal epidermal barrier functions and skin homeostasis in mice. ${ }^{21}$ Based on evidence suggesting a homeostatic role of keratinocyte-intrinsic RABGEF1, we have speculated that this protein could also act as a regulator of homeostasis and inflammation at other epithelial surfaces, such as the intestine. In that site, colonization by microorganisms

\footnotetext{
${ }^{1}$ Laboratory of Immunophysiology, GIGA Institute, Liege University, Liège, Belgium; ${ }^{2}$ Faculty of Veterinary Medicine, Liege University, Liège, Belgium; ${ }^{3}$ Laboratory of Cellular and

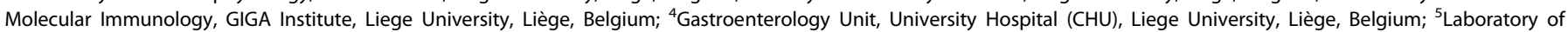

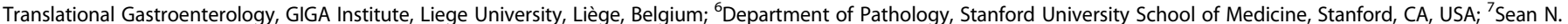

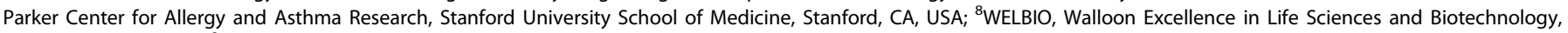
Wallonia, Belgium and ${ }^{9}$ Department of Microbiology and Immunology, Stanford University School of Medicine, Stanford, CA, USA

Correspondence: Thomas Marichal (t.marichal@uliege.be)

These authors contributed equally: Sophie El Abbas, Coraline Radermecker.
}

Received: 5 December 2018 Revised: 18 September 2019 Accepted: 28 September 2019

Published online: 18 October 2019 
a

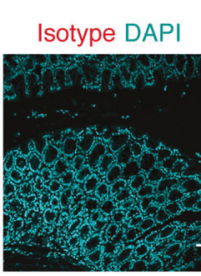

C
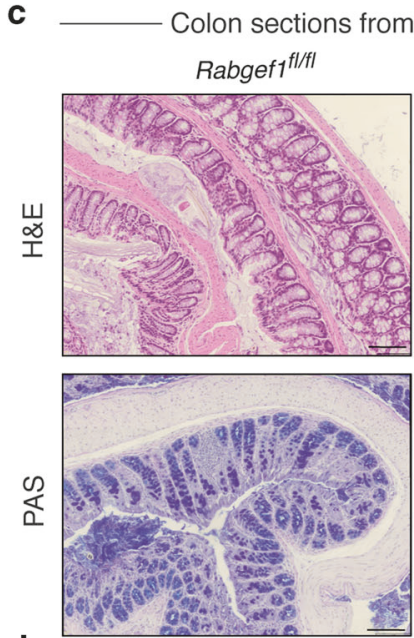

d

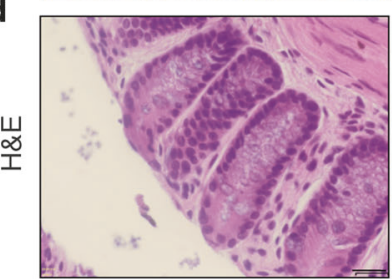

RABGEF1 DAPI

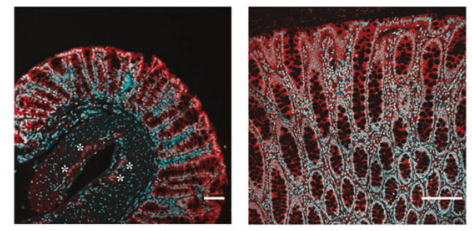

12-month-old mice
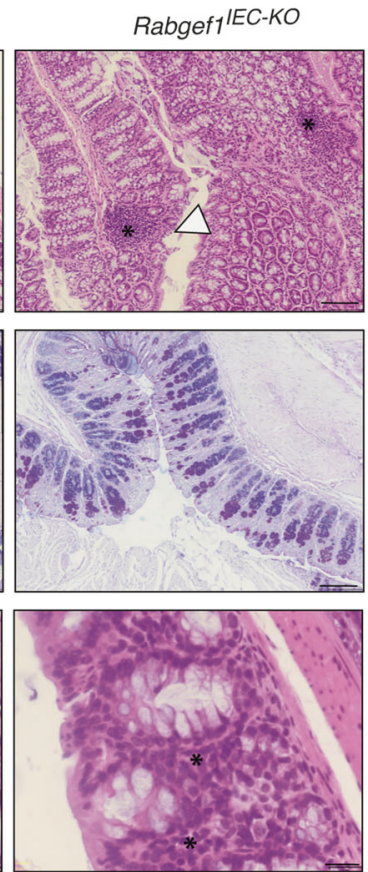

b

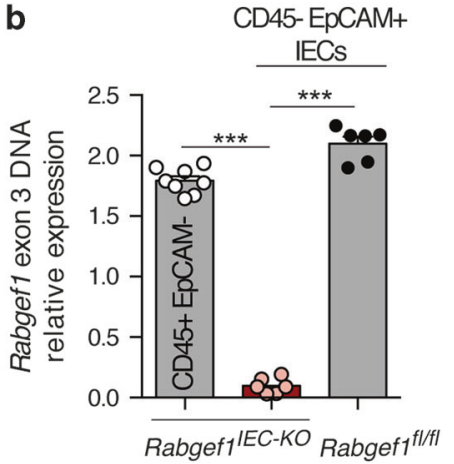

e

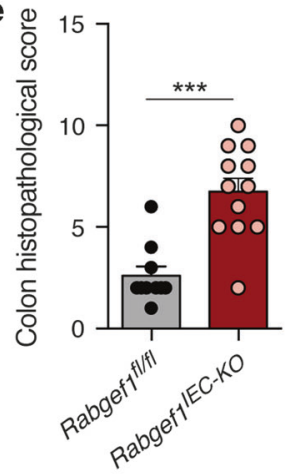

Fig. 1 Spontaneous colitis development in aged C57BL/6 mice with conditional depletion of Rabgef1 in IECs (i.e., Rabgef1 IEC-KO mice). a Representative RABGEF1 staining of colon sections from 8-week-old WT C57BL/6 mice. Pictures are representative of 1 of $>6$ mice analyzed, each of which gave similar results. b Efficiency and specificity of Cre-mediated Rabgef1 exon 3 deletion assessed by quantitative PCR in $\mathrm{CD}^{+} 5^{+} \mathrm{EpCAM}^{-}$leukocytes and CD45 ${ }^{-} \mathrm{EpCAM}{ }^{+}$IECs. c, d Representative hematoxylin \& eosin (H\&E, top panels) and Periodic Acid Schiff (PAS, bottom panels) stainings of colon sections from 12-month-old Rabgef $1^{I E C-K O}$ and littermate controls. Asterisks indicate inflammatory cell infiltrates and arrowhead shows eroded epithelial areas. e Colon histopathological score $(n=10-12$ mice/group). b, e Data are shown as mean + SEM, as well as individual values, and are pooled from $2(\mathbf{b})$ or $>3(\mathbf{e})$ independent experiments. $P$ values were calculated using a oneway ANOVA with Tukey's post hoc tests (b) and a Mann-Whitney test (e). ${ }^{* * *} P<0.001$. Scale bars: $100 \mu \mathrm{m}(\mathbf{a}, \mathbf{c}) ; 20 \mu \mathrm{m}(\mathbf{d})$. WT wild-type

requires tight regulation of the intestinal epithelium in order to cope with the myriad of microbial stimuli and antigens.

\section{RESULTS}

IEC-specific Rabgef1 deletion promotes a spontaneous and delayed colitis

In the mouse colon, RABGEF1 protein was found to be highly expressed in IECs (Fig. 1a) as well as in discrete cell clusters located in the submucosa, which may correspond to the myenteric plexus (Fig. 1a, asterisks). To investigate the functions of IEC-intrinsic RABGEF1 in vivo, Villin-Cre ${ }^{+}$; Rabgef ${ }^{f / f l}$ mice with conditional Rabgef1 deletion within IECs were generated (called Rabgef1 ${ }^{I E C-K O}$ mice hereafter) (Fig. 1b). Under conventional conditions of housing, Rabgef1 ${ }^{I E C-K O}$ mice were born with a Mendelian ratio and did not develop any mortality (Supplementary Fig. 1a), weight loss (Supplementary Fig. 1b) or macroscopic intestinal abnormalities (Supplementary Fig. 1c) until the age of 12 months. Histopathological analyses of colon sections from 10-week-old mice did not reveal development of intestinal pathology in Rabgef1 $1^{I E C-K O}$ mice (Supplementary Fig. 1d, e). However, similar analyses performed on 12-month-old mice showed signs of moderate inflammation (i.e., mucosal and submucosal inflammatory infiltrates) and epithelial damage (i.e., hyperplasia and erosion without goblet cell loss) in the colon of Rabgef IEC-KO mice as compared to controls (Fig. 1c-e), while no abnormalities were found in the small intestine (Supplementary Fig. 2a-c). We also analyzed other organs of aged Rabgef1 ${ }^{I E C-K O}$ mice and did not find any obvious histological abnormalities (Supplementary Fig. 2d-l).

Epithelial RABGEF1 deficiency results in IEC-specific alteration of cytokine expression without systemic effects

Next, we characterized IEC-intrinsic immune and barrier functions and features of systemic immune activation in Rabgeff ${ }^{I E C-K O}$ and littermate Rabgef $f^{f / f l}$ mice. Quantitative reverse transcription polymerase chain reaction (RT-qPCR) analyses of IECs from 12month-old mice revealed that RABGEF1-deficient IECs exhibited a higher expression of $\mathrm{Tnfa}, \mathrm{CCl} 2, \mathrm{CxCl} 1$, and $\mathrm{CxCl} 2$ as compared to the littermate counterparts, while the expression of other cytokines (Il6, Vegfa, CCl11, and CCl5) and Muc2 (coding for Mucin 2) was not affected by RABGEF1 deficiency (Fig. 2a). Such effects were however not observed in IECs from 10-weekold Rabgef1 $1^{I E C-K O}$ mice (Supplementary Fig. 3). To evaluate intestinal permeability to luminal contents, plasma levels of fluorescein isothiocyanate (FITC) were measured following oral 
a

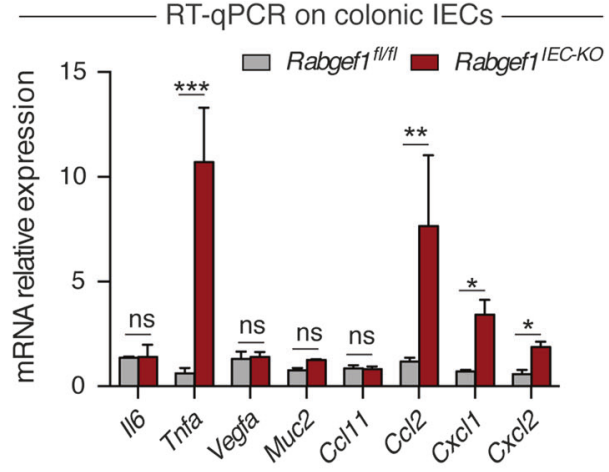

b

Intestinal permeability to FITC dextran

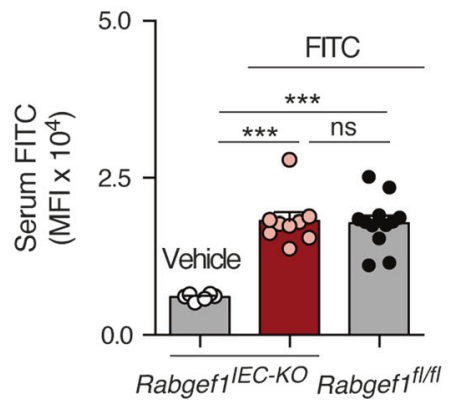

C

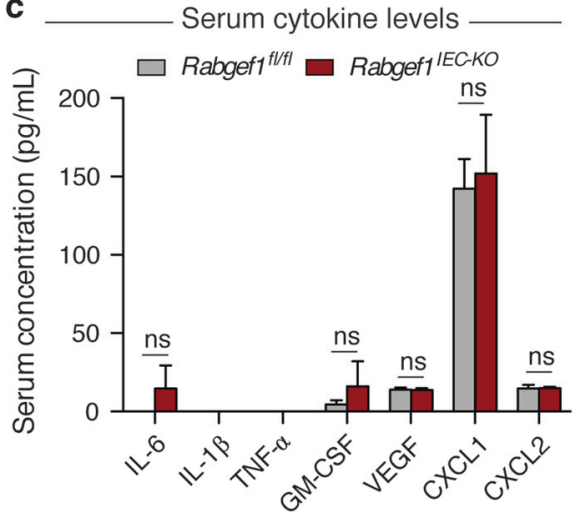

Fig. 2 Assessment of IEC-specific immune and barrier functions and systemic inflammation in 12-month-old Rabgef $1^{I E C-K O}$ mice. a IECs were isolated from Rabgef1 ${ }^{I E C-K O}$ and littermate control mice and expression of the indicated genes was assessed by RT-qPCR ( $n=$ 4 mice/group). b Quantification of intestinal permeability: serum levels of FITC fluorescence $4 \mathrm{~h}$ after FITC-dextran gastric gavage $(n=$ 6-12 mice/group). c Cytokine concentrations measured by Luminex assay in the sera of mice ( $n=7-8$ mice/group). Concentration values below the detection threshold are depicted as zero values. Data are shown as mean + SEM and are pooled from 2 independent experiments, each of them giving similar results. $P$ values were

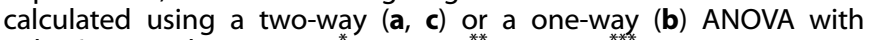
Tukey's post hoc tests. ${ }^{*} P<0.05 ;{ }^{* *} P<0.01 ;{ }^{* * * *} P<0.001$; ns not significant $(P>0.05)$. FITC fluorescein isothiocyanate

administration of FITC-dextran. Notably, no differences were found between 12-month-old Rabgef $1^{I E C-K O}$ and littermate controls (Fig. 2b). Third, serum levels of several cytokines were not altered by IEC-restricted RABGEF1 deficiency in such mice (Fig. 2c). These results indicate that epithelial RABGEF1 deficiency was associated with local changes in IEC innate responses, while no evidence of impaired intestinal permeability or systemic inflammation were observed.
Epithelial RABGEF1 limits colon inflammation and pathology in IL10-deficient mice

The genetic model of colitis based on IL-10 deficiency has been extensively used to help dissecting the mechanisms of IBD. ${ }^{22}$ It is thought to influence IEC responses ${ }^{23-25}$ and promotes a spontaneous, microbiota-dependent colitis. ${ }^{26,27}$ To characterize the effects of IL-10 deficiency on IECs in our housing conditions, we performed mRNA-sequencing (RNA-seq) of FACS-sorted colonic IECs isolated from 10 -week-old $1110^{-/-}$and littermate $1 / 10^{+/+}$mice and found that IECs clustered according to their genotype (i.e., $1 / 10^{-/-}$or $1 / 10^{+/+}$mice) (Supplementary Fig. $4 \mathrm{a}, \mathrm{b}$ ). In addition, we found a significant enrichment, among genes upregulated in $1 / 10^{-1-}$ IECs, of transcripts implicated in oxidative phosphorylation, protein translation and export, and ribosome function (Supplementary Fig. 4c, d), as well as MHC class II-related genes, chemokines, and genes implicated in the response to lipopolysaccharide (LPS) and the negative regulation of inflammation (Supplementary Fig. 4e). Conversely, genes downregulated in $1 / 10^{-1-}$ IECs were shown to be involved in adherens/apical junctions, digestion or transmembrane transporter activity (Supplementary Fig. 4c, d). These results support the idea that IECs isolated from $1110^{-\prime-}$ mice exhibit increased metabolic and innate immune activities associated with impaired barrier and digestive functions.

In order to address the contribution of IEC-intrinsic RABGEF1 in IL-10 deficiency-induced intestinal inflammation, Rabgef1 ${ }^{I E C-K O}$ mice were generated on the $1 / 10^{-/-}$background, and the development of intestinal pathology was evaluated. At the age of 3-4 months, the weight and the colon lengths were not significantly different between $1 / 10^{-/-}$; Rabgef1 $1^{I E C-K O}$ and control $\| 10^{-/-}$; Rabgeff $f^{f / f l}$ mice (Supplementary Fig. 5). However, $\| 10^{-/-}$; Rabgef1 $1^{I E C-K O}$ mice exhibited exacerbated signs of colon inflammation and damage as compared to controls (Fig. 3). Indeed, microscopic analysis of colon sections revealed that $1110^{-1-}$; Rabgef1 ${ }^{I E C-K O}$ mice had more severe inflammation (associated with transmural and dense inflammatory infiltrates), as well as more epithelial damage, sometimes developing into extensive ulcerated areas (Fig. 3a). Immunofluorescent staining of neutrophils with anti-myeloperoxydase (MPO) antibodies showed a higher number of neutrophils infiltrating the colon of $1 / 10^{-/-}$; Rabgef $1^{I E C-K O}$ mice as compared to controls (Fig. 3b, c). In addition, a substantial and consistent loss of mucus-producing goblet cells was observed (Fig. 3d). As a consequence, a higher histopathological score was observed in the colon of $1110^{-/-}$; Rabgef $1^{I E C-K O}$ mice vs. controls (Fig. 3e).

Within IECs, RABGEF1 deficiency was associated with increased expression of genes coding for pro-inflammatory cytokines (II6, Tnfa, Vegfa, CCl11, CCl2, Cxcl1, and $C x \mathrm{Cl}$ ) and reduced expression of the Muc2 gene, consistent with the loss of goblet cells (Fig. 4a). In addition, like the situation observed in 12-monthold Rabgef1 $1^{I E C-K O}$ and littermates, intestinal permeability to FITCdextran and systemic levels of cytokines were not affected by RABGEF1 deficiency in this model (Fig. 4b, c).

Epithelial RABGEF1 regulates susceptibility to DSS-induced colon damage and pathology

Next, we assessed whether IEC-intrinsic RABGEF1 expression could be involved in the regulation of intestinal inflammation triggered by exogenous stimuli such as DSS. We used a model based on administration of $3 \%$ DSS in drinking water for 7 days, which promoted acute increases in numbers of neutrophils, eosinophils, monocytes/macrophages, and dendritic cells as compared to the baseline, a profile that was not observed in 10 -week-old $/ 110^{-/-}$ mice (Supplementary Fig. 6). Despite these differences between the two models, the transcriptomic profile of DSS-exposed colonic IECs evaluated by RNA-seq was strikingly similar to the one of IECs isolated from age-matched $/ 110^{-/-}$animals (Supplementary Fig. 7). 

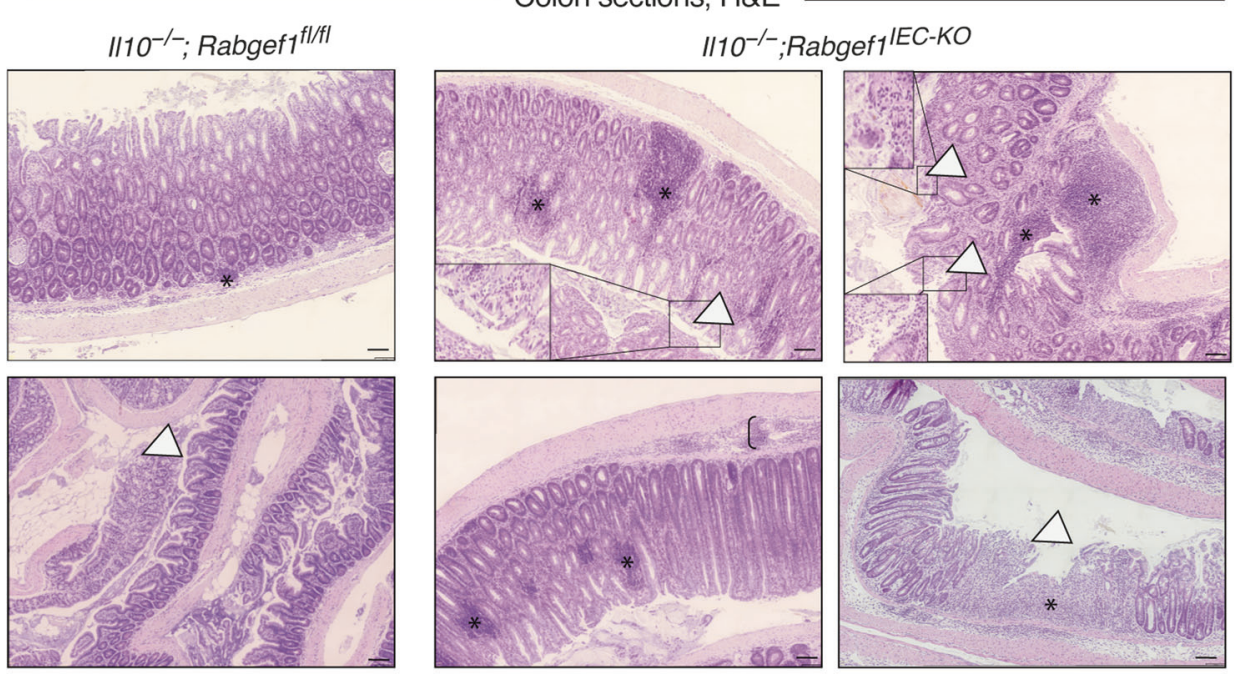

b

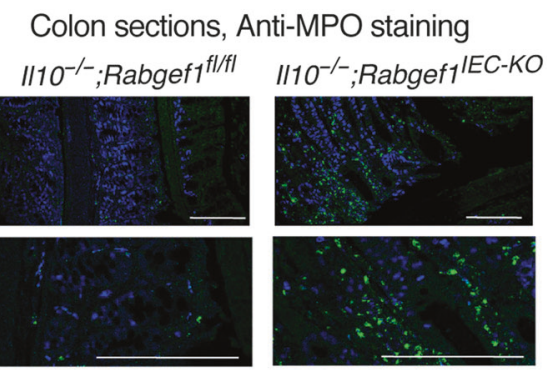

$\square I / 10^{-/-} ;$Rabgef1 ${ }^{\text {flffI }} \square I 110^{-/-} ;$Rabgef1 IEC-KO

c Average $\mathrm{MPO}^{+}$cells/field
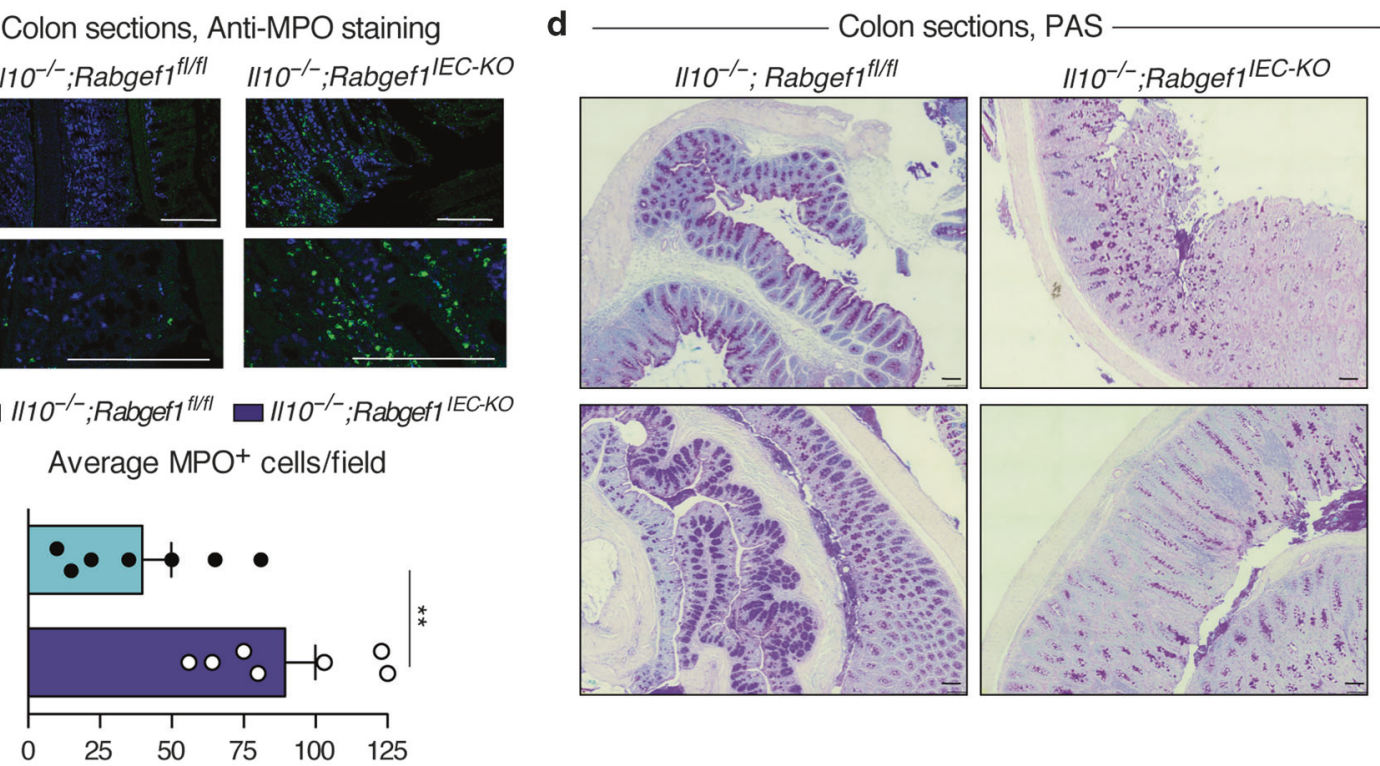

e

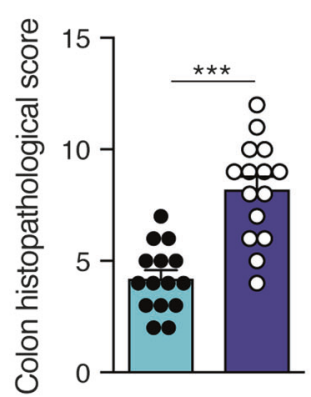

Fig. 3 Contribution of epithelial RABGEF1 to the regulation of intestinal pathology in IL-10-deficient mice. Comparisons between 3- and 4month-old Rabgef1 ${ }^{I E C-K O}$ and Rabgef1 $7^{f / f l}$ (control) mice raised on the $/ 110^{-/-}$background are shown. a Representative hematoxylin \& eosin (H\&E) staining of colon sections. Asterisks indicate focal inflammatory cell infiltrates, arrowheads show, at higher power, ulcerated epithelial areas, and brackets show areas of severe transmural inflammation. b Representative confocal microscopy pictures of myeloperoxidase (MPOgreen) staining of colon sections. DAPI ${ }^{+}$nuclei are blue. c Quantification of average MPO ${ }^{+}$cells per field $(n=7$ mice/group). d Representative Periodic Acid Schiff (PAS) staining of colon sections. e Colon histopathological score ( $n=15$ mice/group). Data are shown as mean + SEM, as well as individual values, and are pooled from $2(\mathbf{c})$ or $>3(\mathbf{e})$ independent experiments. $P$ values were calculated by a Mann-Whitney test ${ }^{* *} P<$ $0.01 ;{ }^{* * *} P<0.001$. Scale bars: $100 \mu \mathrm{m}$ 
a

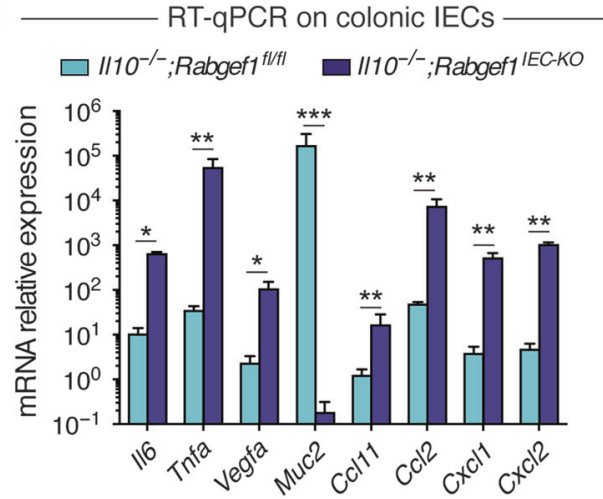

b

Intestinal permeability

to FITC dextran

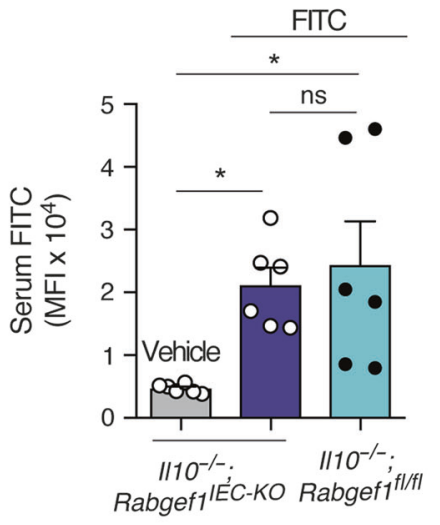

c

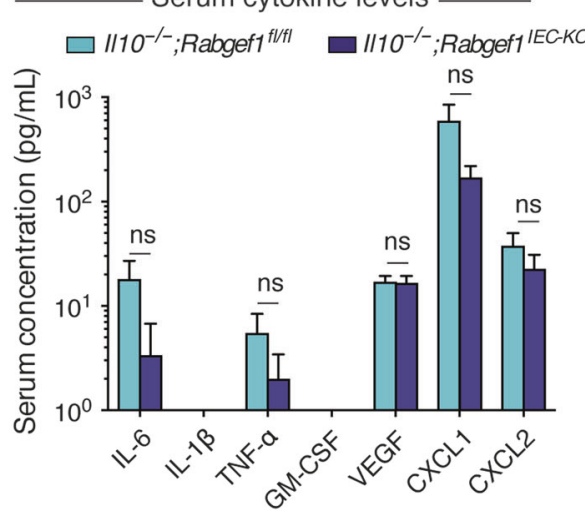

Fig. 4 Contribution of epithelial RABGEF1 to the regulation of IEC innate responses, intestinal permeability, and systemic inflammation in IL-10-deficient mice. Comparisons between 3-to-4-month-old Rabgef $1^{I E C-K O}$ and Rabgef1 $1^{f / f l}$ (control) mice raised on the $1110^{-/-}$ background are shown. a IECs were isolated and expression of the indicated genes was assessed by RT-qPCR ( $n=4$ mice/group). b Quantification of intestinal permeability: serum levels of FITC fluorescence $4 \mathrm{~h}$ after FITC-dextran gastric gavage ( $n=6$ mice/ group). c Cytokine concentrations measured by Luminex assay in the sera of mice ( $n=6-7$ mice/group). Concentration values below the detection threshold are depicted as zero values. Data are shown as mean + SEM, as well as individual values in (g), and are pooled from two independent experiments, each of them giving similar results. $P$ values were calculated by a two-way $(\mathbf{a}, \mathbf{c})$ or a one-way (b) ANOVA with Tukey's post hoc tests. ${ }^{*} P<0.05$; ${ }^{* *} P<0.01$; ${ }^{* * *} P<$ 0.001 ; ns not significant $(P>0.05)$. FITC fluorescein isothiocyanate
Based on such findings, we decided to evaluate the contribution of epithelial RABGEF1 using this model.

Compared to Rabgef1 $1^{f / 7}$ mice, Rabgef1 $1^{\text {IEC-KO }}$ mice exhibited liquid, sticky and bloody feces (Fig. 5a) and significantly shorter and highly inflamed colons (Fig. 5b, c). Histopathological examination of colons from DSS-treated Rabgef1 $1^{I E C-K O}$ mice revealed increased epithelial hyperplasia and crypt elongation, the presence of dense granulocyte-rich cell infiltrates in the submucosa, and extensive epithelial ulceration and transmural inflammation when compared to DSS-treated controls (Fig. 5d, e), resulting in a more severe histopathological score (Fig. 5f). Two days after DSS treatment, when intestinal permeability to FITCdextran is known to be affected by $\mathrm{DSS}^{28}$ permeability to FITCdextran was, again, not different between Rabgef1 $1^{\mid E C-K O}$ and control mice (Fig. $5 \mathrm{~g}$ ). These results indicate that, similar to its role in the IL-10 deficiency model, RABGEF1 expression is also critical in limiting DSS-induced inflammation and damage. Altogether, our results so far support the idea that RABGEF1 deficiency promotes development of intestinal inflammation and locally exacerbates IEC innate responses.

RABGEF1 modulates intrinsic expression of genes implicated in IEC physiology, endocytosis, and innate immunity

To gain mechanistic insights into RABGEF1-dependent regulation of IEC responses, we performed RNA-seq analyses of FACS-sorted IECs from 10 -week-old $/ 110^{-/-} ;$Rabgef $1^{I E C-K O}$ and littermate $\| 10^{-/-}$; Rabgef $1^{f / f l}$ mice. As shown in Fig. 6a, RABGEF1-deficient IECs were separated from the control counterparts across principal component (PC)1 (explaining $40 \%$ of the variance between samples). Using an adjusted $P$ value $\left(P_{\text {adj }}\right)<10^{-2}$, a total of 102 genes were found to be differentially expressed (DE) between $1 / 10^{-/-}$; Rabgef1 $1^{I E C-K O}$ and $1 / 10^{-/-}$; Rabgef1 ${ }^{f / f l}$ IECs (Fig. $6 \mathrm{~b}$ and Supplementary Table 1).

First, on the basis of MouseNet v2 network ${ }^{29}$ and a bibliographical search, we found that several genes implicated in transmembrane transporter activity and metabolic processing of nutrients were downregulated in RABGEF1-deficient IECs (Fig. 6C), supporting the idea that IECs' physiological functions were impaired when RABGEF1 was absent. Conversely, genes implicated in the regulation of innate immune pathways, such as the MAPK pathways or the NF-KB pathway, were upregulated in RABGEF1-deficient IECs (Fig. 6c).

Second, gene set enrichment analysis (GSEA), used to identify biological signatures in IECs from $/ 110^{-/-}$; Rabgef $1^{I E C-K O}$ mice as compared to those from $1110^{-/-}$; Rabgef $f^{f / f l}$ mice, revealed a significant enrichment, among genes downregulated in RABGEF1-deficient IECs, of genes involved in endocytic events (Fig. $6 \mathrm{~d}$ ). We also assessed the gene expression profiles of IECs from Rabgef $1^{I E C-K O}$ and littermate control mice on the wild-type (WT) background by RNA-seq (Supplementary Fig. 8a, b and Supplementary Table 2). Notably, we found a highly significant similarity in gene expression profiles of IECs from Rabgef1 $7^{I E C-K O}$ mice under the IL-10-deficient and those under the WT background (Supplementary Fig. 8c), as well as a downregulation of endocytosis-related transcripts in IECs from Rabgef $1^{I E C-K O}$ mice as compared to the control counterparts (Supplementary Fig. 8d). These data support the conclusion that IECs exhibit alterations of their endolysosomal system when RABGEF1 is absent.

RABGEF1-deficient IECs display endosomal abnormalities in vitro To support our RNA-seq-based observations at the protein level, we took advantage of immortalized mouse IECs, which display structural features of primary IECs and can functionally respond to microbial stimulation. ${ }^{30}$ Lentiviral-based expression of RABGEF1 knockdown was induced in IECs (Supplementary Figs. 9 and 10). Using antibodies directed against the RABGEF1 endocytic effector Rab5, we showed that the distribution of Rab5 staining at steady- 
a

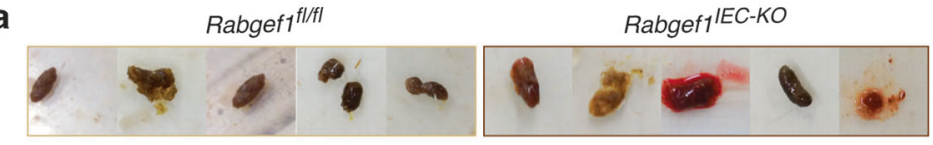

b

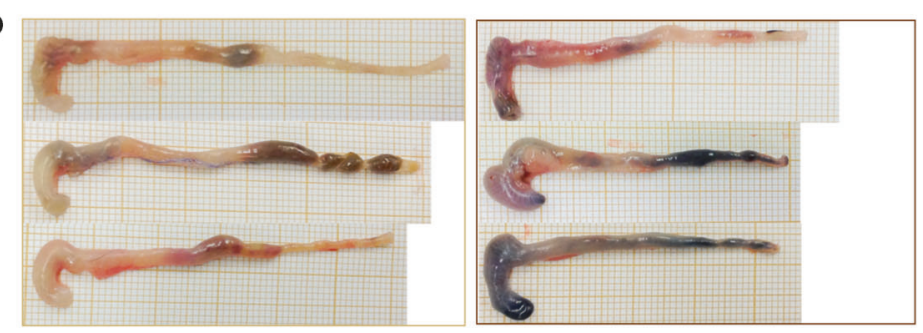

C

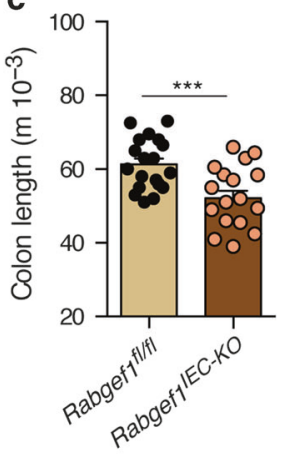

d
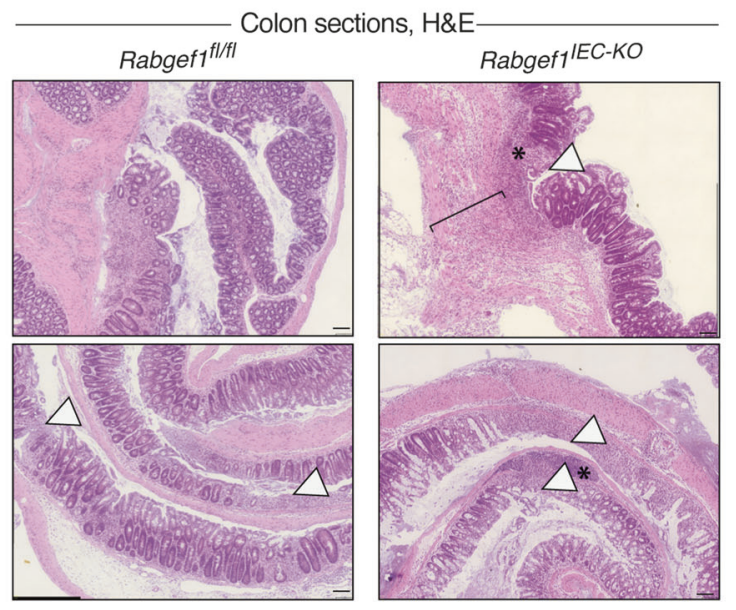

f

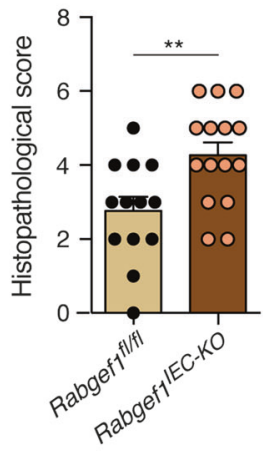

e

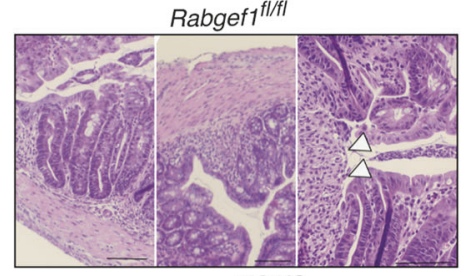

Rabgef1 IEC-KO

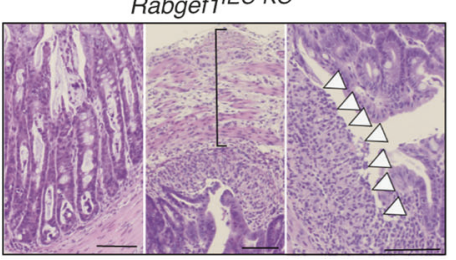

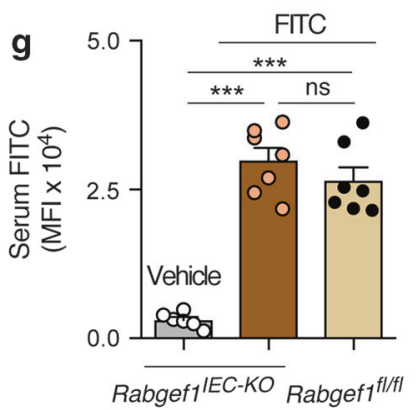

Rabgef1 ${ }^{I E C-K O}$ Rabgefi ${ }^{\text {fl|fl }}$

Fig. 5 Contribution of epithelial RABGEF1 to DSS-induced inflammation and damage. a-f Comparisons between 8-to-12-week-old female Rabgef $1^{I E C-K O}$ and Rabgef $1^{f / f}$ littermate control mice after 7 days of DSS exposure ( $3 \%$ in drinking water) are shown. a, b Photographs of feces (a) and ceaca and colons (b) 7 days after DSS treatment. Pictures are representative of 3 of $>15$ mice analyzed, each of them giving similar results. c Colon length ( $n=17-18$ mice/group). d, e Representative H\&E staining of colon sections at lower (d) and higher (e) power. Asterisks indicate focal inflammatory cell infiltrates, arrowheads show ulcerated areas of the epithelium, and the bracket encompass areas of severe transmural inflammation. f Histopathological score assessed in the colon ( $n=13-15$ mice/group). g Quantification of intestinal permeability 2 days after DSS treatment: serum levels of FITC fluorescence $4 \mathrm{~h}$ after FITC-dextran gastric gavage $(n=6-7$ mice/group). $\mathbf{c}, \mathbf{f}, \mathbf{g}$ Data are shown as mean + SEM, as well as individual values, and are pooled from $3(\mathbf{c}, \mathbf{f})$ or $2(\mathbf{g})$ independent experiments. $P$ values were calculated by a twotailed unpaired Student's $t$ test (c), a Mann-Whitney test (f) or a one-way ANOVA with Tukey's post hoc tests $(\mathbf{g}){ }^{*{ }^{* *}} P<0.01$; $^{* * *} P<0.001 ;$ ns not significant $(P>0.05)$. Scale bars: $100 \mu \mathrm{m}$. FITC fluorescein isothiocyanate

state was markedly different between RABGEF1-sufficient vs. RABGEF1-deficient IECs, with Rab5 being more diffusely distributed and less structurally organized when RABGEF1 was absent (Fig. 7a). After 60 min of LPS stimulation of RABGEF1-sufficient IECs, Rab5 staining intensity was increased and concentrated in polarized areas of the cell (Fig. 7a). Conversely, Rab5 staining remained more diffuse and less polarized in LPS-stimulated RABGEF1-deficient IECs (Fig. 7a).
In addition, we assessed the levels of early endosome antigen-1 (EEA-1) protein in RABGEF1-sufficient and RABGEF1-deficient IECs by Western blotting. EEA-1 is an early endosomal marker and effector of Rab5 whose levels have been shown to be reduced in Rab5-deficient cells ${ }^{17}$. We found that EEA-1 levels significantly increased following LPS stimulation of RABGEF1-sufficient IECS, supporting the occurrence of early endosomal events following LPS stimulation (Fig. 7b, c). By contrast, we found that EEA-1 levels 
RNA-seq analysis of IECs sorted from 10 -week-old $1 / 10^{-/-}$mice

a $\square \| 10^{-/-} ;$Rabgeff $1^{f l|f|} \square \| 10^{-/-} ;$Rabgef1 ${ }^{I E C-K O}$

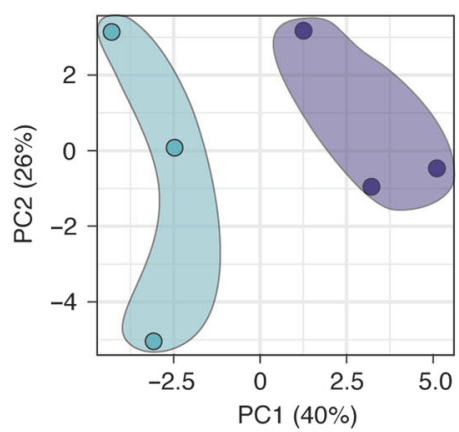

b

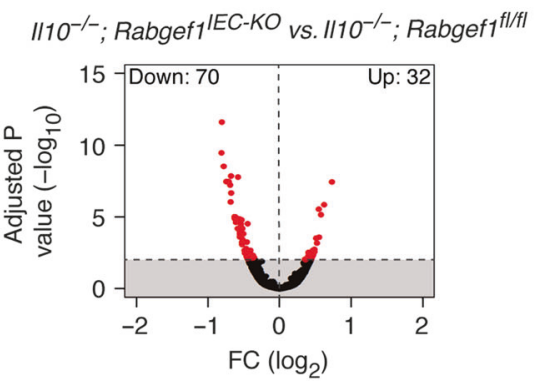
GSEA analysis between
$\| 110^{-/-} ;$Rabgef $1 / E C-K O \& \| 1 / 0^{-/-} ;$Rabgef $1^{f|f|} \mid \mathrm{ECs}$

d and defined gene sets

\begin{tabular}{ccc}
\hline Gene sets & NES & FDR \\
\hline
\end{tabular}

Endosome organization

Enriched in $1 / 10^{-1-}$;

Rabgef $1^{f|f| f \mid} \mid \mathrm{ECS}$

0.032 ransporter
activity

Clcn2

Recycling of

Recycling of

membrane

-1.5 Enriched in $1 / 10^{-/}$;

Rabgef $1^{f|f|} \mid \mathrm{ECS}$

0.105

\begin{tabular}{l|l} 
Cyp2d34 & Metabolic \\
Hexb & processes
\end{tabular}

Hexb

Ctss

Upp1

Wnt5a

Cd44 Reg. MAPK

Rgs2 pathway

Cd24a Reg. NF-kB

Tnfrsf19] $\begin{gathered}\text { Reg. NF-kB } \\ \text { pathway }\end{gathered}$

Relative

expression

Low

\begin{tabular}{|c|c|}
\hline $\begin{array}{l}\text { Endo- } \\
\text { Iysosome }\end{array}$ & $\begin{array}{c}\text { Enriched in } \| 10^{-1-} \\
\text { Rabgeff }\end{array}$ \\
\hline $\begin{array}{c}\text { Endosomal } \\
\text { part }\end{array}$ & $\begin{array}{c}\text { Enriched in } 1 / 10^{-/-} \\
\text {Rabgef }^{f|l / f|}{ }_{\text {IECs }}\end{array}$ \\
\hline
\end{tabular}

0.113

0.158

Fig. 6 RNA-seq analyses of colonic IECs isolated from 10 -week-old $\| 110^{-/-}$; Rabgef1 $1^{I E C-K O}$ and $/ 110^{-/-}$; Rabgef ${ }^{f / f l}$ mice. a Two-dimensional PCA comparing RABGEF1-deficient (Rabgef1 $1^{I E C-K O}$ ) and RABGEF1-sufficient (Rabgef $\left.1^{f / f}\right)$ IECs from $/ / 10^{-1-}$ mice. Percentages indicate the variability explained by each component. $n=3$ replicates/group, each replicate representing a pool of IECs isolated from three mice. $\mathbf{b}$ Volcano plot depicting the DE genes between RABGEF1-deficient and RABGEF1-sufficient IECs from $1 / 10^{-/-}$mice. DE genes with a $P_{\text {adj }}$ value $<10^{-2}$ are colored in red. c Heatmaps showing examples of significantly DE genes within individual samples. Genes are grouped according to their

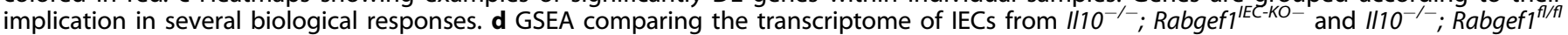
mice with defined gene sets. Circle size is proportional to the normalized enrichment score (NES), whose numeric value is also provided. The false-discovery rates (FDR) are also indicated

did not increase after LPS stimulation in RABGEF1-deficient IECs (Fig. 7b, c). Altogether, these data indicate that Rab5-dependent endocytic events occur in IECs after LPS stimulation but that RABGEF1 deficiency disrupts such endocytic events.

RABGEF1 regulates IEC-intrinsic p38-dependent signaling and chemokine production

Next, we examined the signaling pathways mediating the interaction of LPS and the Toll like receptor (TLR)4. Upon LPS stimulation, RABGEF1 knockdown had no effects on the levels of phosphorylation of $\mathrm{I} \mathrm{KBa}$, an inhibitor of NF-KB whose phosphorylation is required for NF-KB activation (Fig. 8a, b). ${ }^{31}$ While increased levels of phosphorylated ERK were noted in RABGEF1 knockdown IECs, such activation effect was not restored in RABGEF1-rescued IECs (Fig. 8a, b). However, after 5 or 15 min of LPS stimulation, RABGEF1 knockdown IECs exhibited increased phosphorylation levels of p38 MAPK as compared to those in RABGEF1-sufficient IECs, a signaling activation event that was not observed when RABGEF1 expression was restored (Fig. 8a, b).
Functionally, such activation was associated with an increased ability of RABGEF1 knockdown IECs to produce the chemokines CCL2, CCL11, CXCL1, and CXCL2 as compared to the control or RABGEF1-rescued IECs (Fig. 8c). Altogether, these data support the idea that RABGEF1 influences the TLR4-mediated p38 MAPKdependent signaling pathways and their associated productions of several chemokines in IECs.

Intestinal inflammation in Rabgef1 ${ }^{\text {IEC-KO }}$ mice is mediated by intrinsic MYD88-dependent signaling and microbe-derived signals MYD88 mediates the signaling responses induced by activation of IL-1/IL-18/Toll receptor superfamily members to induce activation of NF-KB and MAPK pathways leading to the release of proinflammatory mediators. ${ }^{32}$ Since RABGEF1 regulates the p38 MAPK pathway in response to LPS in vitro, which signals through MYD88, we wondered whether MYD88 signaling contributed to the phenotype observed in aged Rabgef $1^{I E C-K O}$ mice. In order to test this, we generated and analyzed Rabgef1/Myd $88^{I E C-K O}$ mice, in which IECs lack both MYD88 and RABGEF1. Notably, deletion of 
a

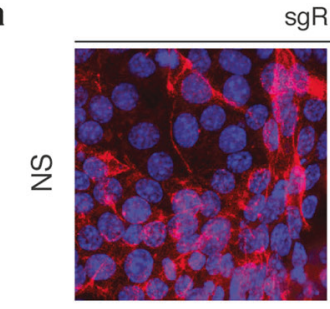

sgRNA NT
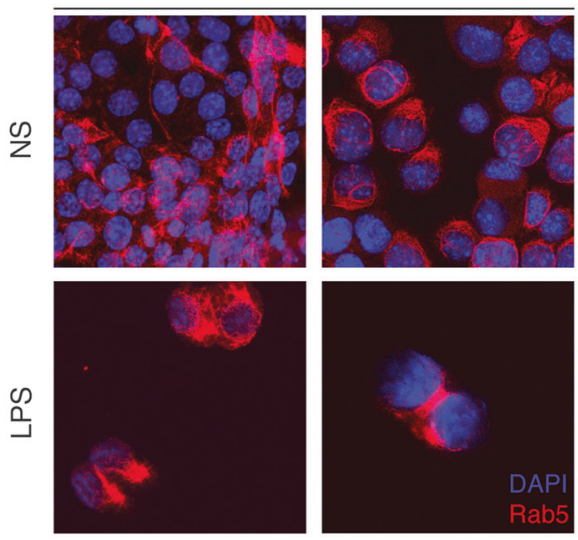

sgRNA Rabgef1
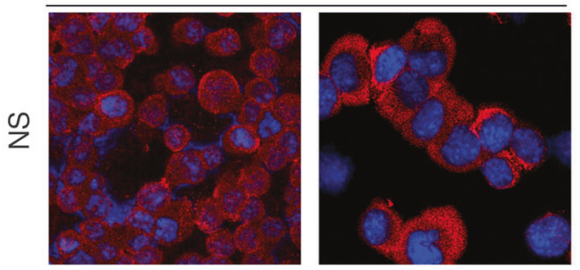

$\stackrel{\infty}{\square}$
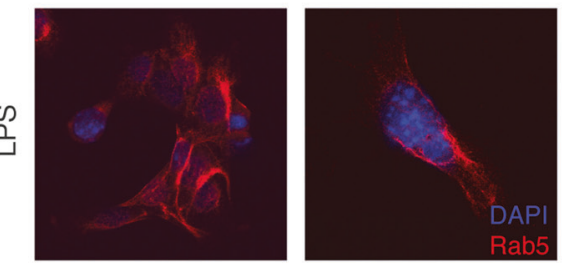

b

Minutes after LPS stimulation (100 $\mathrm{ng} / \mathrm{mL})$ sgRNA NT $\quad$ sgRNA Rabgef1

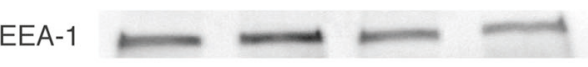

HSP90

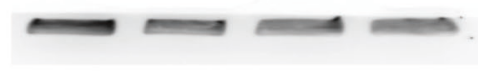

C

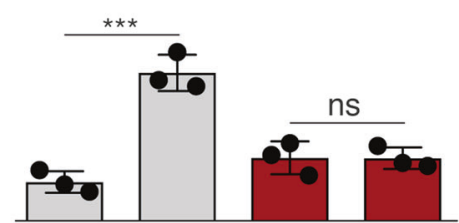

EEA-1

Fig. 7 Endosomal abnormalities in RABGEF1-deficient IECs in vitro. Immortalized primary mouse IECs transduced with lentiviral plasmids encoding nontarget (NT) and Rabgef1 single guide (sg) RNA and Cas9 (sgRNA NT and sgRNA Rabgef1, respectively) were used. a High-resolution confocal microscopy pictures of Rab5 (red) and DAPI (blue) staining in IECs, at steady-state and $1 \mathrm{~h}$ after LPS stimulation $(100 \mathrm{ng} / \mathrm{ml})$. Pictures are representative of similar results obtained in each of two experiments performed with two different batches of cells. b Representative Western Blots and c protein quantification showing the levels of EEA-1 0 and 60 min after LPS stimulation. Data are shown as mean \pm SEM, and are pooled from independent experiments performed with three separate batches of cells. $P$ values were calculated using a one-way ANOVA test followed by Tukey's post hoc tests for multiple comparisons. ${ }^{* * *} P<0.001$; ns not significant $(P>0.05)$

Myd88 in Rabgef1/Myd88 $8^{I E C-K O}$ mice largely prevented the colitis observed in Rabgef1 $1^{I E C-K O}$ counterparts (Fig. 9a, b), which was associated with a similar IEC cytokine expression profile between Rabgef1/Myd88 ${ }^{I E C-K O}$ mice and littermate controls (Fig. 9c). These results support the conclusion that MYD88-dependent signaling mediates intestinal inflammation in Rabgef $1^{I E C-K O}$ mice.

The commensal microorganisms that colonize the colon after birth are critical components of host physiology and immune homeostasis in healthy individuals, but can also contribute to pathological processes in a dysregulated system ${ }^{1,33,34}$. IECs express a wide range of TLRs, which sense and transduce microbiota-derived signals via MYD88, except for TLR3 ${ }^{4,7,31}$. Since the delayed colitis of Rabgef1 $1^{I E C-K O}$ mice was dependent on MYD88, we sought to determine the contribution of the microbiome in this context.

First, we wondered whether IEC-specific RABGEF1 depletion could induce alterations in the colon microbial composition. Therefore, we performed sequencing of bacterial $16 \mathrm{~S}$ ribosomal RNA genes (16S rRNA-seq) to compare the composition of the microbiota found in the colon lumen of Rabgef1 $1^{I E C-K O}$ and littermate Rabgef $1^{f / f l}$ controls at the age of 10 months. Multidimensional scaling and unsupervised hierarchical clustering did not reveal any genotype-dependent clustering (Fig. 10a, b), and bacterial diversity was found to be similar in the colon of Rabgef1 ${ }^{I E C-K O}$ as compared to the control counterparts (Fig. 10c). In addition, colon microbiota composition was not different between Rabgef1 $1^{I E C-K O}$ and Rabgef1 $1^{f / / f l}$ control mice (Fig. 10d), supporting that epithelial RABGEF1 deficiency does not induce changes in the gut bacterial composition.

Second, we continuously fed 6-month-old Rabgef $1^{\text {IEC-KO }}$ and littermate controls with autoclaved water with or without antibiotics $(A B)$ for a period of 4 months, and colon histopathological scoring was performed at the end of the treatment. Notably, we found that antibiotic treatment significantly reduced the features of colitis and the histopathological score in ABtreated Rabgeff ${ }^{I E C-K O}$ mice as compared to the untreated counterparts (Fig. 10e, f), supporting the conclusion that microbiotadependent signals are involved in the delayed colitis that develops in aged Rabgef1 $1^{I E C-K O}$ mice.

\section{DISCUSSION}

We have identified herein a novel role for the guanine exchange factor RABGEF1 in the regulation of IEC functions and intestinal inflammation in vivo. Using mice conditionally deficient for Rabgef1 in IECs, we have provided experimental evidence that epithelial RABGEF1 deficiency promotes development of a microbiota- and MYD88-dependent colitis with age. Such colitis was associated with an upregulation of IEC-intrinsic transcripts coding for pro-inflammatory cytokines and chemokines. Notably, however, no defects in intestinal barrier permeability, nor features of systemic immune activation, were observed in 12-month-old Rabgef1 ${ }^{I E C-K O}$ mice. This supports the idea that epithelial RABGEF1 regulates events that are restricted to the gut mucosa, without substantial systemic effects. Interestingly, it has been reported in some mouse models that intestinal and systemic pathology could be regulated by distinct mechanisms and disconnected from each other. $^{35}$

To investigate the role of epithelial RABGEF1 in the context of intestinal inflammation, we used two distinct models of colitis, initiated either by a dysregulated host immune response (i.e., IL-10 deficiency) or by an epithelial-eroding agent (i.e., DSS). The fact that IECs from 10 -week-old $/ 110^{-1-}$ mice or from DSS-exposed WT mice shared a strikingly similar profile supports the conclusion that the IEC response to colitogenic triggers, whether they are endogenous (i.e., IL-10 deficiency) or exogenous (i.e., DSS), is conserved. This is particularly interesting, since the IL-10 deficient and the DSS models are different in several aspects, e.g., chronic vs. acute, and endogenous immune trigger vs. exogenous trigger, respectively. 
a

Minutes after LPS stimulation (100 $\mathrm{ng} / \mathrm{mL})$

sgRNA NT sgRNA Rabgef1 Rescued Rabgef1

p-p38

$\begin{array}{llllllllllll}0 & 5 & 15 & 60 & 0 & 5 & 15 & 60 & 0 & 5 & 15 & 60\end{array}$

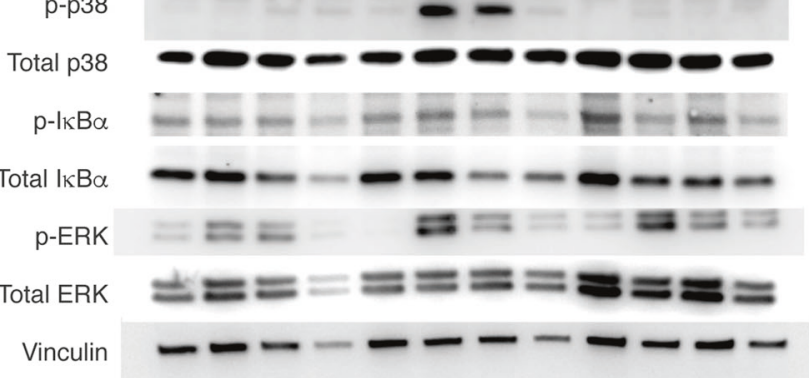

b

Minutes after LPS stimulation (100 $\mathrm{ng} / \mathrm{mL}$ )

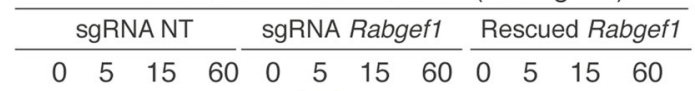
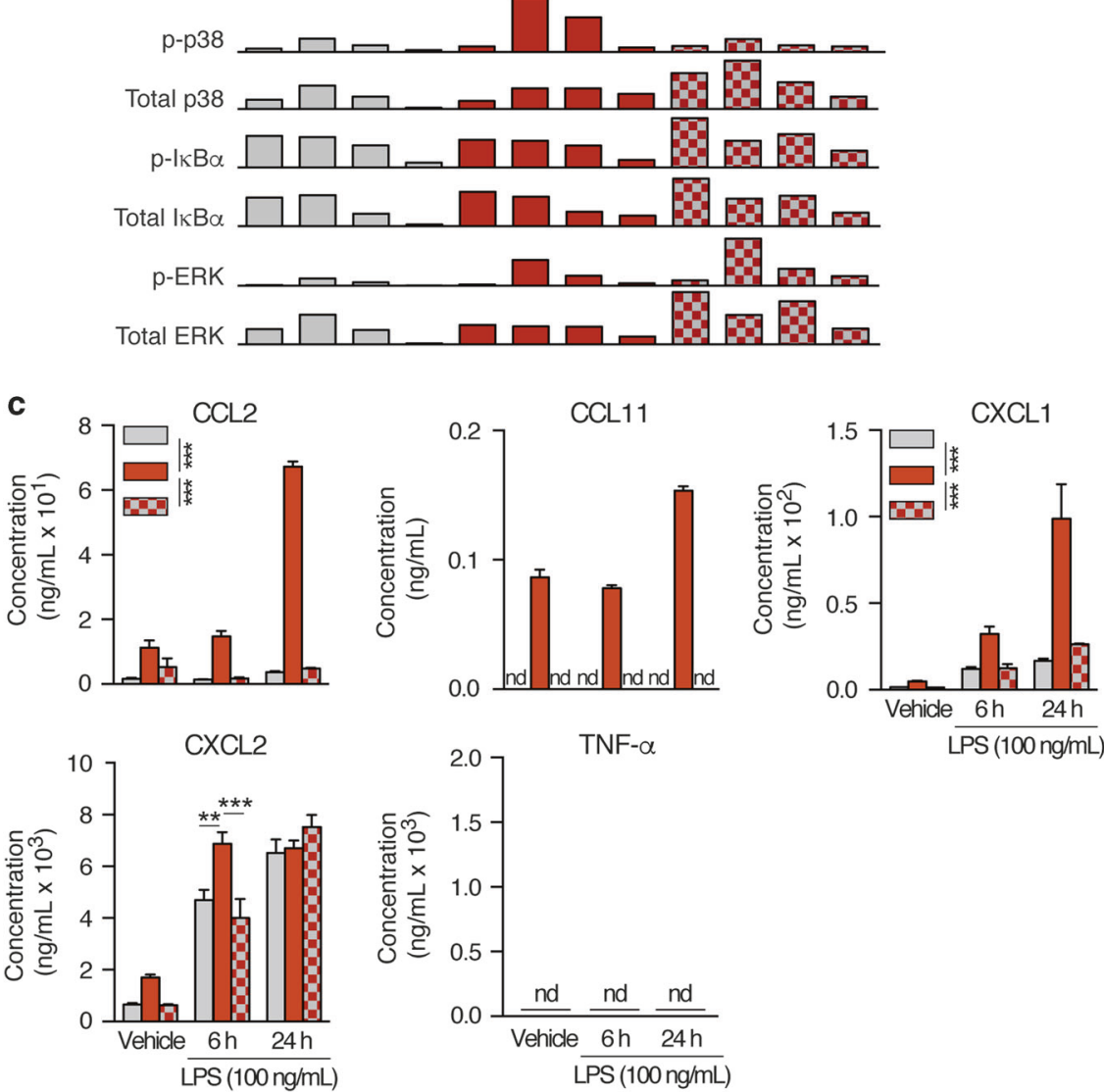

$\overline{\mathrm{LPS}(100 \mathrm{ng} / \mathrm{mL})}$

Fig. 8 RABGEF-dependent regulation of p38-dependent signaling and chemokine production in IECs. Immortalized primary mouse IECs transduced with lentiviral plasmids encoding nontarget (NT) and Rabgef1 single guide (sg)RNA and Cas9 (sgRNA NT and sgRNA Rabgef1, respectively), and RABGEF1-deficient IECs (sgRNA Rabgef1) transduced with plasmids encoding Rabgef1 to restore RABGEF1 expression (Rescued RABGEF1) were used. a Representative Western Blots and b protein quantification showing the levels of the indicated phosphoproteins and total proteins at different times following LPS stimulation. Results are representative of similar results obtained in each of three experiments performed with three separate batches of cells. c Cytokine concentrations measured by Luminex assay in the supernatants of media (Vehicle)- or LPS-stimulated IECs. Data are mean + SEM and are pooled from two independent stimulation experiments performed with two separate batches of cells. $P$ values were calculated using a two-way ANOVA test followed by Tukey's post hoc tests for multiple comparisons. ${ }^{* *} P<0.01 ;{ }^{* * *} P<0.001$; nd not detected

Given the differences observed in the recruitment of immune cells between the two models, it seems likely that the eroding properties of DSS and the subsequent release of damageassociated signals from dying epithelial cells initially contribute to the acute immune cell infiltration upon DSS exposure, as opposed to the situation in IL-10-deficient mice. However, the features of DSS-induced pathology, including the cellular immune profile in the colon, are thought to be influenced by many factors, including the dose and duration of DSS treatment, the gender and genetic background of the host, and the microbial status or the 


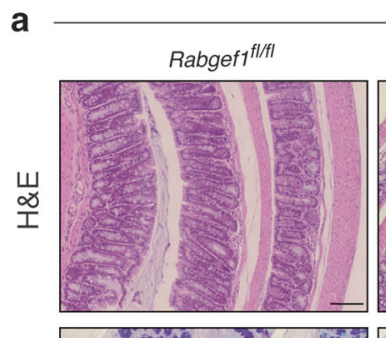

Colon sections from 6-8-month-old mice
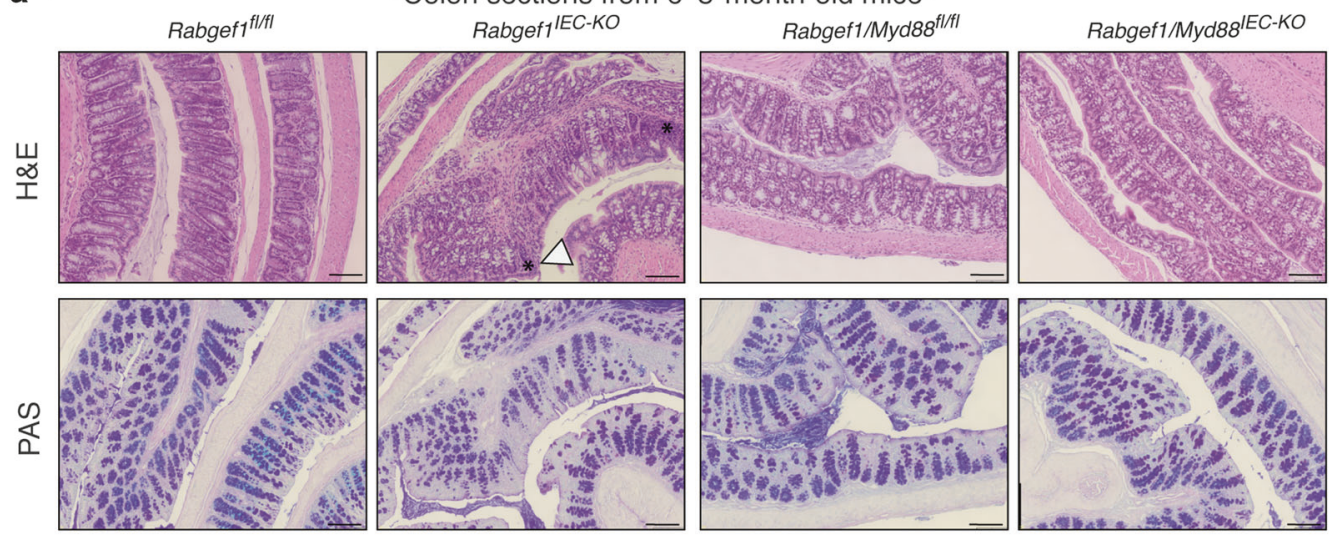

b

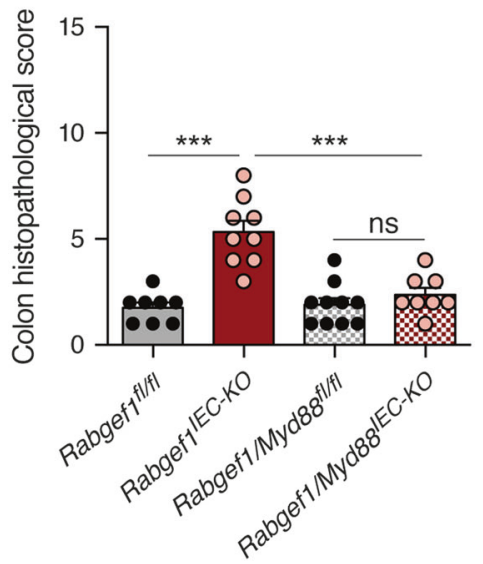

C

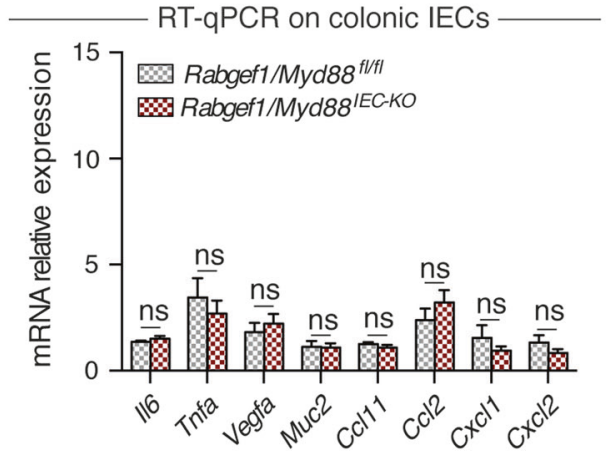

Fig. 9 Role of IEC-intrinsic MYD88 signaling in the development of intestinal inflammation in Rabgef1 $1^{I E C-K O}$ mice. Analyses were performed on 6-to-8-month-old mice. a Representative H\&E and PAS staining of colon sections. Asterisks indicate focal inflammatory cell infiltrates and arrowhead shows eroded epithelial areas. b Colon histopathological score $(n=8-10$ mice/group). c IECs were isolated from Rabgef1/ $M y d 88^{I E C-K O}$ and littermate control mice and expression of the indicated genes was assessed by RT-qPCR ( $n=4$ mice/group). b, c Data are shown as mean + SEM, as well as individual values in (b), and are pooled from two independent experiments. $P$ values were calculated using a one-way (b) or a two-way (c) ANOVA with Tukey's post hoc tests. ${ }^{* * *} P<0.001$; ns not significant $(P>0.05)$. Scale bars: $100 \mu \mathrm{m}$

housing conditions, which makes comparisons between studies difficult. $^{36,37}$

During either form of colitis, IEC-specific RABGEF1 deficiency exacerbated several cardinal features of colon pathology and was associated with increased expression of pro-inflammatory cytokines, but did not impact intestinal permeability and systemic levels of pro-inflammatory cytokines, supporting the existence of RABGEF1-dependent local regulatory mechanisms. Moreover, genes involved in the regulation of the endolysosomal system were found to be downregulated in IECs from Rabgef1 ${ }^{I E C-K O}$ mice as compared to controls, both under the $1110^{-1-}$ and the WT background. This suggests that the GEF activity of RABGEF1 for the GTPase Rab5, critical for the biogenesis of endosomes ${ }^{17}$, mediates such effects in IECs in vivo.

The transcriptomic analyses of RABGEF1-deficient IECs also suggested a possible involvement of RABGEF1 in regulating MAPK- or NF-KB-dependent innate signaling. Specifically, we used an in vitro tool to investigate the potential RABGEF1-dependent regulation of such pathways. Immortalized mouse primary IECs were chosen because they allow analysis of innate immune functions ${ }^{30}$, but circumvent the restricted availability and homogeneity of primary cells and organoids, respectively, and also avoid the structural and functional alterations often present in intestinal cell lines. Using this model, we have shown on the one hand that RABGEF1 knockdown markedly altered Rab5-dependent endocytic events, at steady-state and upon LPS stimulation. On the other hand, we found that, upon LPS stimulation,
RABGEF1 specifically prevented activation of p38 MAPK and downregulated the production of several chemokines, including CCL2, CXCL1, and CXCL2, consistent with the transcriptomic profile of RABGEF1-deficient IECs in vivo.

Notably, RABGEF1-sufficient IECs seemed particularly hyporesponsive to LPS stimulation in our experimental setting, which reflects well the hypo-responsiveness of IECs to LPS and other TLR ligands in vivo. ${ }^{38-40}$ However, when IEC-intrinsic RABGEF1 expression was knocked down, LPS responsiveness was potentiated, leading to aberrant activation of the p38 MAPK and increased chemokine production. These data are consistent with the hypothesis that RABGEF1 may act as a regulator of IEC innate responsiveness in vivo, and that such responsiveness is compromised when epithelial RABGEF1 is absent, which would contribute to increased chemokine expression and the exaggerated inflammation observed in Rabgef1 ${ }^{I E C-K O}$ mice. In this regard, it is interesting to note that the monocyte chemoattractant CCL2 and the neutrophil chemoattractants CXCL1 and CXCL2 are thought to be involved in human or experimental IBD, ${ }^{41-44}$ and the p38 MAPK pathway has been shown to specifically control the expression of chemokines within IECs. ${ }^{45,46}$

The delayed development of colitis in Rabgef1 $1^{I E C-K O}$ mice suggested that environmental factors may mediate disease susceptibility in those animals, and microbe-derived signals arguably represented appealing candidates. Indeed, Rabgef1 $1^{I E C-K O}$ mice developed intestinal inflammation at a site of heavy microbial exposure (i.e., the colon) but did not have detectable 


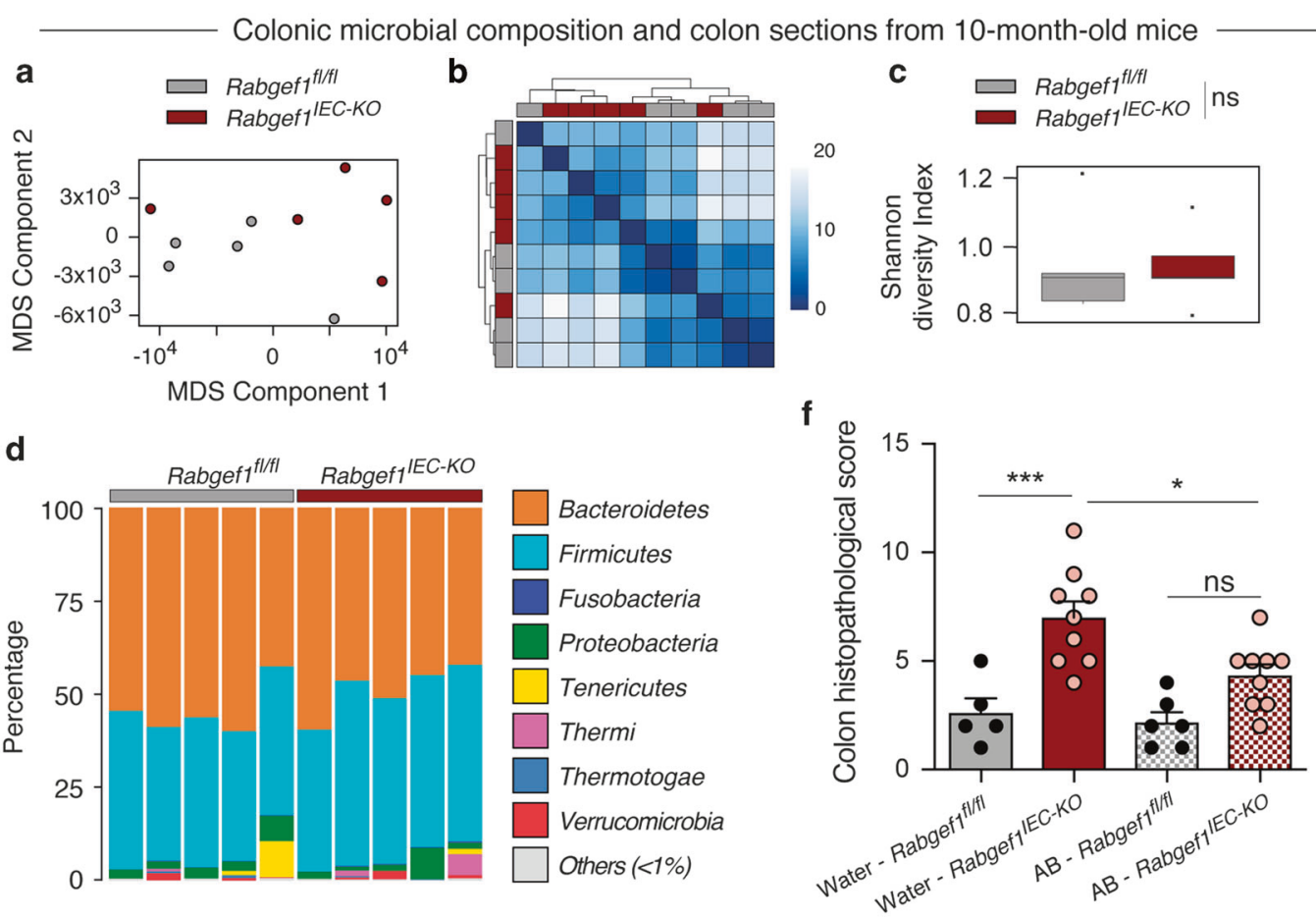

e

e Water - Rabgeft $1^{f / H}$

Water - Rabgef1 $1 E C-K O$
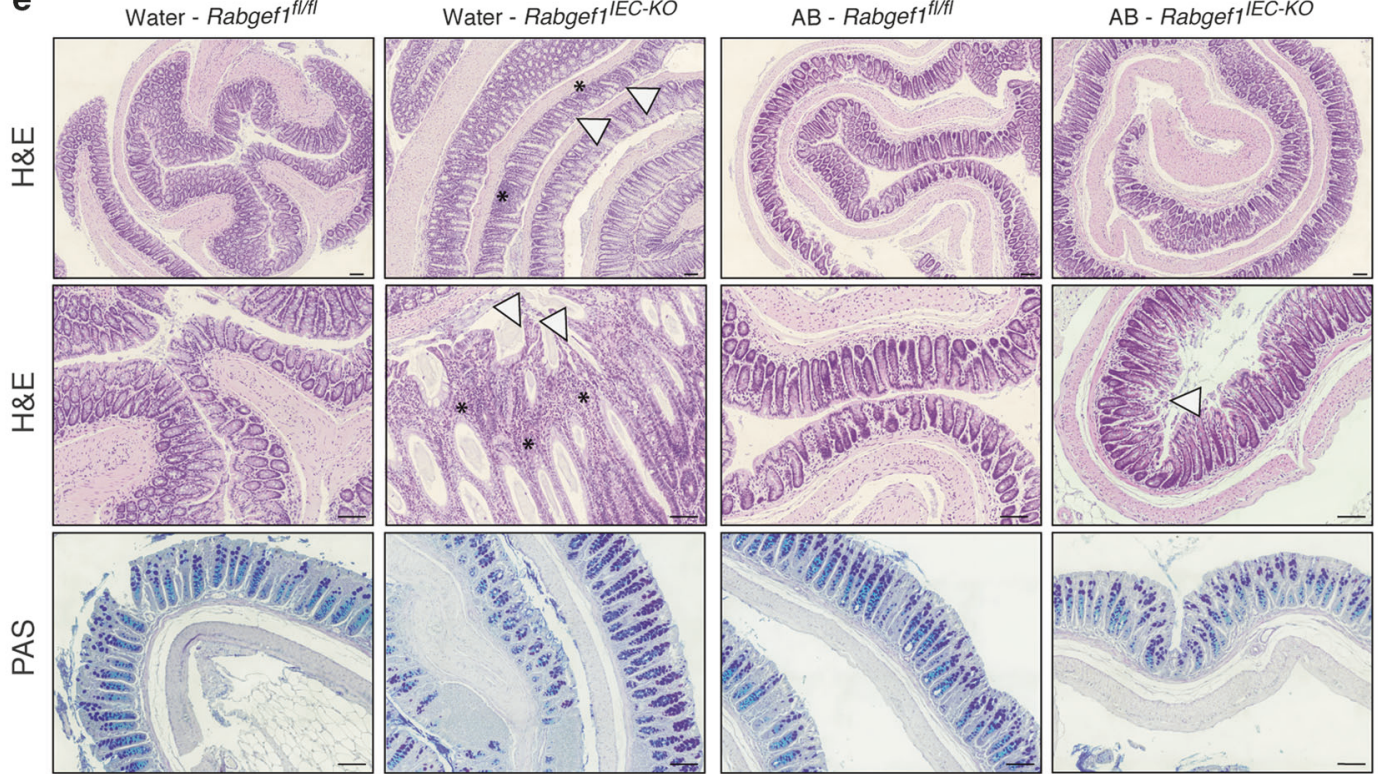

Fig. 10 Influence of the gut microbiome of Rabgef1 ${ }^{I E C-K O}$ mice on the delayed development of colon pathology. a-d Microbial composition of colonic luminal contents in 10-month-old Rabgef1 ${ }^{1 E C-K O}$ and littermate control mice. 16S rRNA-seq and taxonomic comparisons were performed between Rabgef ${ }^{I E C-K O}$ and littermate control mice ( $n=5$ mice/group) at the phylum level. a Multi-dimensional scaling (MDS) graphs. b Unsupervised hierarchical clustering of biological replicates. The color scale reflects the clustering distance $(0=$ highest correlation; $20=$ the lowest correlation) between 16S rRNA expression patterns of two samples c Shannon diversity indexes. d Percentage of the most represented taxons $(>1 \%)$ within each individual sample, presented as stacked bars. e Representative H\&E and PAS staining of colon sections. Stars indicate focal inflammatory cell infiltrates and arrowhead shows eroded epithelial areas. $\mathbf{f}$ Colon histopathological score $(n=5-9$ mice/ group). Data are shown as mean + SEM, as well as individual values, and are pooled from two independent experiments. $P$ values were calculated using a one-way ANOVA with Tukey's post hoc tests. ${ }^{*} P<0.05 ;{ }^{* * * *} P<0.001$; ns not significant $(P>0.05)$. Scale bars: $100 \mu \mathrm{m}$

pathology in the small intestine where the bacterial load is much lower. Moreover, we showed that the spontaneous colitis of Rabgef1 $1^{I E C-K O}$ mice was MYD88-dependent, and it is well-known that many microbe-associated patterns can activate TLRs through the adapter protein MYD88 ${ }^{47,48}$. By analyzing the microbial composition of the colons of Rabgef $1^{I E C-K O}$ mice and by treating them with $A B$, we have provided evidence that the intestinal inflammation that develops in aged Rabgef $1^{I E C-K O}$ mice depends on microbe-derived commensal signals. In younger mice, it may be that regulatory mechanisms are more effective in preventing development of colitis.

Previous work supports the conclusion that RABGEF1 can regulate many different pathways of innate immunity. In mast cells in vitro, RABGEF1 has been shown to regulate FceRI-dependent activation of 
Ras and ERK $^{20}$ and SCF-dependent activation of ERK and JNK. ${ }^{49}$ In the skin, we have demonstrated that keratinocyte RABGEF1 can maintain skin homeostasis by regulating intrinsic IL1R-MYD88-NFKB-dependent signaling pathways independently of the skin microbiota. ${ }^{21}$ The current study indicates that epithelial RABGEF1 can also regulate microbiota- and MYD88-dependent intestinal inflammation and p38 MAPK-dependent signaling and chemokine production in IECs. We cannot exclude the possibility that IL-1 $\beta$ contributes to MYD88-dependent inflammation in Rabgef1 $1 E C-K O$ mice. However, RABGEF1-dependent regulatory pathways appear to exhibit differences in functional and interactive properties in epidermal keratinocytes, IECs and mast cells, suggesting that there may be tissue- and cell-specific mechanisms mediating different RABGEF1 effects.

The precise mechanisms by which the activities of RABGEF1 regulate the activation of innate immune pathways, and the expression of cytokines, in IECs are still unclear. Nevertheless, our data are in accord with the hypothesis that the GEF activity for Rab5 may contribute to these effects. Indeed, we have demonstrated that deletion of RABGEF1 can markedly alter Rab5dependent endocytic events, as well as TLR4-MYD88-dependent signaling pathways, in IECs. Since activation of TLR4 by LPS is regulated by endosomal-mediated endocytosis, which requires Rab5 activity that is regulated by RABGEF1 ${ }^{13-17,48}$, RABGEF1 deficiency may downregulate LPS-induced receptor-mediated endocytosis. Because RABGEF1 represents an important negative regulator of TLR4 signaling, RABGEF1 deficiency may promote the increased activation of LPS-dependent pathways, resulting in an increased cytokine expression in IECs.

Stimulation of the TLR4-MYD88-dependent pathway by LPS also can activate several transcription factors, including NF-KB, AP-1, and $\mathrm{CREB}^{31,48}$. In our signal transduction analyses, we showed that stimulation of RABGEF1-deficient IECs with LPS did not increase levels of $\mathrm{p}-\mathrm{I} \mathrm{KBa}$, suggesting that the NF-KB pathway is not involved in the observed effect elicited by RABGEF1 deficiency. Similarly, our signaling studies also showed that ERK MAPK was not involved in the LPS-induced effect observed with these cells, suggesting that the AP-1 pathway is likewise not directly involved. However, levels of phosphorylation of p38 MAPK were significantly enhanced by LPS stimulation in RABGEF1-deficient IECs, suggesting that the CREB transcription factor pathway is involved in mediating the cytokine transcription effects during the TLR4dependent activation of $\mathrm{IECs}^{31,48}$. Obviously, future detailed mechanistic studies using epidermal keratinocytes, mast cells and IECs expressing different RABGEF1 domain mutants will help in investigating how different domains of RABGEF1 are involved in such effects and the precise molecular mechanisms underlying the regulatory functions of RABGEF1.

In conclusion, our study identifies a previously unknown function for RABGEF1 protein in the regulation of MYD88dependent intestinal inflammation and IEC innate pathways in mice. It is currently unknown whether dysregulated epithelial RABGEF1 expression also contributes to intestinal pathology in IBD in humans. However, in 2013, Montero-Meléndez and colleagues performed microarray gene expression profiling of colon biopsies from a small cohort of IBD patients in search of predictive genomic signatures, and identified RABGEF1 transcript level as a clinically relevant predictor for classifying IBD patients, with glucocorticoid-resistant patients exhibiting the lowest RABGEF1 expression. ${ }^{50}$ Together with our study, these data suggest that RABGEF1-dependent regulatory mechanisms may represent promising therapeutic targets in the context of IBD.

\section{METHODS}

Mice

C57BL/6 Rabgef1 ${ }^{f / f l}$ mice were previously described. ${ }^{21}$ Transgenic C57BL/6 Villin-Cre (B6.Cg-Tg(Vil1-cre)997Gum/J), ${ }^{51} \quad / 10^{-/-}$

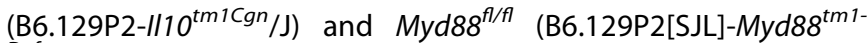
107

Defr $/ J$ ) mice were from The Jackson Laboratory (Bar Harbor, USA). Mice were housed under specific pathogen free (SPF) conditions and maintained in a 12-h light-dark cycle with food and water ad libitum. Age-matched littermate mice were used for experiments throughout the study. All animal experiments described in this study were carried out with the approval of the Institutional Animal Care and Use Committee of Liege University. The "Guide for the Care and Use of Laboratory Animals", prepared by the Institute of Laboratory Animal Resources, National Research Council, and published by the National Academy Press, as well as European and local legislations, were followed carefully.

Reagents and antibodies

Details about reagents and antibodies can be found in the Supplementary material.

\section{Immunostaining and confocal microscopy}

Paraffin-embedded intestinal sections were deparaffinized, rehydrated, and heat-induced epitope retrieval was performed in sodium citrate buffer. After blocking, samples were incubated overnight at $4{ }^{\circ} \mathrm{C}$ with primary antibodies. Secondary antibody staining was performed at room temperature.

For immunostaining of IECs in vitro, IECs were cultured on slides for $24 \mathrm{~h}$. Slides were methanol-fixed and permeabilized before staining with primary and secondary antibodies.

Images were acquired using a Leica SP5 (Wetzlar, Germany) confocal laser-scanning microscope. Additional Details can be found in the Supplementary material.

Assessment of intestinal permeability

To evaluate intestinal permeability, FITC-dextran (FD4, Merck, Darmstadt, Germany) was administered by gastric gavage to fasting mice ( $4 \mathrm{~h}$ food and water fasting, $0.6 \mathrm{mg} / \mathrm{g}$ diluted in phosphate-buffered saline (PBS)). Four hours later, plasma was collected and plasma levels of FITC were measured by spectrophotometry.

Quantitative RT-PCR on IECs

After IEC FACS sorting, RNA was extracted and reverse-transcribed into CDNA, and PCR reactions were performed in duplicate. Primer sequences can be found in Supplementary Table 3. Data were normalized to the housekeeping genes Hprt and Ppib in the same sample. Additional details can be found in the Supplementary material.

Quantification of serum cytokines

Blood was collected from the tail vein and sera were assayed for CXCL1, CXCL2, GM-CSF, IL1- $\beta$, IL-6, VEGF, and TNF- $\alpha$ using Luminex Mouse Magnetic Assay (R\&D, Minneapolis, USA), according to the manufacturer's recommendations.

Colon histopathological scoring

For evaluation of colon pathology in antibiotic-treated or untreated Rabgef1 ${ }^{I E C-K O}$, Rabgef1/Myd88 $8^{I E C-K O}$ and control counterparts at steady-state, on the WT or $1110^{-/-}$background, the scoring system described in the report of Dieleman et al. ${ }^{52}$ was adapted as described in the Supplementary material.

Mouse model of DSS-induced colitis

Experiments involving DSS exposure involved between 8- and 12week-old female mice. Acute colitis was induced by addition of $3 \%$ DSS (DSS Colitis grade, MP biomedicals, Santa Ana, USA) to the drinking water for 7 days. Stool consistency was evaluated at day 7 during $4 \mathrm{~h}$ and mice were then sacrificed to perform a macroscopic evaluation of the colons and for histopathological analyses. The severity of DSS-induced colitis was evaluated, as 
previously reported ${ }^{53}$ and as described in the Supplementary material.

In vitro IEC culture and stimulation

Mouse CI-mulNTEPI cells (InSCREENeX, Braunschweig, Germany) were cultured in muINTEPI complete medium (InSCREENeX). Plates were precoated with collagen solution (InSCREENeX) for $2 \mathrm{~h}$ and washed with PBS before use. When cultured cells reached $80 \%$ confluence, NT control, RABGEF1-knockdown and RABGEF1rescued IECs were seeded in 24 -well plates $\left(4 \times 10^{5}\right.$ cells). For confocal microscopy analysis, cells were cultured and stimulated as described above. For Western blot studies, cells were stimulated with LPS $(100 \mathrm{ng} / \mathrm{ml})$ for 5,15 , or $60 \mathrm{~min}$; for cytokine measurements, cells were stimulated with LPS for 6 or $24 \mathrm{~h}$ and cell culture supernatants were assayed for CCL2, CCL11, CXCL1, CXCL2, and TNF-a using Luminex Mouse Magnetic Assay (R\&D, Minneapolis, USA).

\section{Western blotting}

Mouse IECs were lysed in lysis Buffer (3.03 g TRIS pH $7.5+0.19 \mathrm{~g}$ $\mathrm{EDTA}+5 \mathrm{~mL} \mathrm{NP}-40+6 \mathrm{~g} \mathrm{NaCl}$ in $500 \mathrm{~mL}$ DQ water) supplemented with Complete ${ }^{\mathrm{T}}$ Protease Inhibitor Cocktail (Roche, Basel, Switzerland) and PhosSTOP ${ }^{T m}$ Phosphatase Inhibitor Cocktail (Roche, Basel, Switzerland). Protein concentrations in the resulting lysates were measured using the Pierce ${ }^{T m}$ BCA Protein Assay Kit (ThermoFisher Scientific, Waltham, USA). Totally, $50 \mu \mathrm{g}$ proteins were loaded in 4-20\% Mini-PROTEAN ${ }^{\circledast}$ TGX $^{\text {Tm }}$ Gel (Bio-rad, Hercules, USA) and electroblotted onto Invitrolon polyvinylidene fluoride membranes (Novex, ThermoFisher Scientific, Waltham, USA). Membranes were blocked in $5 \%$ nonfat dry milk in Tris-buffered saline-Tween $20(0.1 \%)$ buffer and then probed with primary antibodies in 5\% bovine serum albumin-tris-buffered saline-Tween $20(0.1 \%)$ buffer. Equal loading was confirmed by probing for actin, vinculin or HSP-90a.

\section{Antibiotic treatment}

Six-month-old Rabgef1 $1^{I E C-K O}$ mice and Rabgef1 ${ }^{f / f l}$ littermate controls were fed autoclaved water with or without enrofloxacin 1\% (Baytril, Bayer) for 4 months.

\section{Other methods}

See the Methods section in Supplementary material for cell isolation, staining and flow cytometry, assessment of Rabgef1 deletion by quantitative PCR, sample preparation and histology, high-throughput mRNA-sequencing, GSEA, lentiviral-based RABGEF1 knock down and rescuing of mouse IECs, 16S rRNA gene sequencing and metagenomic analyses.

\section{Statistical analyses}

Respect of the assumptions of normal distribution of residuals and homoscedasticity were verified. Unless otherwise indicated, data are presented as mean + SEM, as well as individual values, from independent experiments and were pooled for analysis in each data panel. Statistical analyses were performed using Prism 6 (GraphPad Software, La Jolla, USA), except for RNA-seq data, for which the R package "DESeq2" was used. We performed the Mantel-Cox test, one-way or two-way ANOVA with Tukey's post hoc tests for multiple comparisons, and Mann-Whitney or unpaired two-tailed Student's $t$ tests as mentioned in the respective figure legends. We considered a $P$ value lower than 0.05 as significant. ${ }^{*} P<0.05 ;{ }^{* *} P<0.01,{ }^{* * *} P<0.001$; ns not significant.

\section{DATA AVAILABILITY}

The RNA-seq data provided in this manuscript have been deposited in the ArrayExpress database at EMBL-EBI (https://www.ebi.ac.uk/arrayexpress/ experiments/E-MTAB-7315) under accession number E-MTAB-7315.

\section{ACKNOWLEDGEMENTS}

We thank all members of the Laboratories of Cellular and Molecular Immunology and Immunophysiology for discussions; Sandra Ormenese, Raafat Stefan, Alexandre Hego, and Jean-Jacques Goval from the GIGA Flow Cytometry and Cell Imaging Platform; Pierre Drion, Gaëlle Lambert and all staff members from the GIGA Mouse facility and Transgenics Platform; Cécile Oury for discussions about colitis induction protocols; Chantal Humblet and staff members from the Immunohistology Platform; Benoît Charloteaux, Manon Deckers, Latifa Karim, and members from the Genomics Platform; Pauline Maréchal, Cedric François, Raja Fares, and Ilham Sbai for their excellent technical and administrative support. The Cellular and Molecular Immunology Laboratory is supported by an Excellence Of Science (EOS) program from the F.R.S.-FNRS and FWO. S.J.G., M.T. and S.-Y.T. are supported by National Institutes of Health grant NIH/NIAMS R01 AR067145. F.B. is supported by the F.R.S.FNRS for the FRFS-WELBIO under grant CR-2012S-01R. T.M. is a Research Associate of the F.R.S.-FNRS and is supported by an "Incentive Grant for Scientific Research" of the F.R.S.-FNRS (F.4508.18), by the FRFS-WELBIO under grant CR-2017s-04, by the Acteria Foundation and by an ERC Starting Grant (ERC-StG-2018 IM-ID 801823).

\section{AUTHOR CONTRIBUTIONS}

T.M. conceived of the project. S.E.A., C.R., D.P., M-A.M., T.A., C.J.D., E.L., S-Y.T., M.T., F.B., S.J.G. and T.M. were involved in experimental design. S.E.A. and C.R. performed most of the experiments and analyzed the data, and compiled the data with the help of T.M. C.B., J.S., M.M. and M-A.M. helped with experiments. Q.B., D.P. and T.M. performed the bioinformatic analyses with the support of the GIGA Genomics Platform. S-Y.T., M.T. and S.J.G. provided Rabgef $f^{f / f l}$ mice. S.E.A. and T.M. prepared the figures. T.M. secured funding and wrote the original paper. All authors participated in writing or editing the paper.

\section{ADDITIONAL INFORMATION}

The online version of this article (https://doi.org/10.1038/s41385-019-0211-z) contains supplementary material, which is available to authorized users.

Competing interests: The authors declare no competing interests.

Ethics statement: All animal experiments described in this study were carried out with the approval of the Institutional Animal Care and Use Committee of Liege University (DE1626). The "Guide for the Care and Use of Laboratory Animals", prepared by the Institute of Laboratory Animal Resources, National Research Council, and published by the National Academy Press, as well as European and local legislations, were followed carefully.

Publisher's note Springer Nature remains neutral with regard to jurisdictional claims in published maps and institutional affiliations.

\section{REFERENCES}

1. Kaiko, G. E. \& Stappenbeck, T. S. Host-microbe interactions shaping the gastrointestinal environment. Trends Immunol. 35, 538-548 (2014).

2. Peterson, L. W. \& Artis, D. Intestinal epithelial cells: regulators of barrier function and immune homeostasis. Nat. Rev. Immunol. 14, 141-153 (2014).

3. Okumura, R. \& Takeda, K. Roles of intestinal epithelial cells in the maintenance of gut homeostasis. Exp. Mol. Med. 49, e338 (2017).

4. McClure, R. \& Massari, P. TLR-dependent human mucosal epithelial cell responses to microbial pathogens. Front. Immunol. 5, 386 (2014).

5. Pastorelli, L., De Salvo, C., Mercado, J. R., Vecchi, M. \& Pizarro, T. T. Central role of the gut epithelial barrier in the pathogenesis of chronic intestinal inflammation: lessons learned from animal models and human genetics. Front. Immunol. 4, 280 (2013).

6. Gallo, R. L. \& Hooper, L. V. Epithelial antimicrobial defence of the skin and intestine. Nat. Rev. Immunol. 12, 503-516 (2012).

7. Rakoff-Nahoum, S., Paglino, J., Eslami-Varzaneh, F., Edberg, S. \& Medzhitov, R. Recognition of commensal microflora by toll-like receptors is required for intestinal homeostasis. Cell 118, 229-241 (2004).

8. Podolsky, D. K. Inflammatory bowel disease. N. Engl. J. Med. 347, 417-429 (2002).

9. Strober, W., Fuss, I. \& Mannon, P. The fundamental basis of inflammatory bowel disease. J. Clin. Invest. 117, 514-521 (2007).

10. Jostins, L. et al. Host-microbe interactions have shaped the genetic architecture of inflammatory bowel disease. Nature 491, 119-124 (2012).

11. Hooper, L. V. \& Gordon, J. I. Commensal host-bacterial relationships in the gut. Science 292, 1115-1118 (2001).

12. Ni, J., Wu, G. D., Albenberg, L. \& Tomov, V. T. Gut microbiota and IBD: causation or correlation? Nat. Rev. Gastroenterol. Hepatol. 14, 573 (2017). 
13. Delprato, A., Merithew, E. \& Lambright, D. G. Structure, exchange determinants, and family-wide rab specificity of the tandem helical bundle and Vps9 domains of Rabex-5. Cell 118, 607-617 (2004).

14. Horiuchi, $H$. et al. A novel Rab5 GDP/GTP exchange factor complexed to Rabaptin-5 links nucleotide exchange to effector recruitment and function. Cell 90, 1149-1159 (1997).

15. Zerial, M. \& McBride, H. Rab proteins as membrane organizers. Nat. Rev. Mol. Cell Biol. 2, 107-117 (2001).

16. Mattera, R. \& Bonifacino, J. S. Ubiquitin binding and conjugation regulate the recruitment of Rabex-5 to early endosomes. EMBO J. 27, 2484-2494 (2008).

17. Zeigerer, A. et al. Rab5 is necessary for the biogenesis of the endolysosomal system in vivo. Nature 485, 465-470 (2012).

18. Lee, $\mathrm{S}$. et al. Structural basis for ubiquitin recognition and autoubiquitination by Rabex-5. Nat. Struct. Mol. Biol. 13, 264-271 (2006).

19. Mattera, R., Tsai, Y. C., Weissman, A. M. \& Bonifacino, J. S. The Rab5 guanine nucleotide exchange factor Rabex-5 binds ubiquitin (Ub) and functions as a Ub ligase through an atypical Ub-interacting motif and a zinc finger domain. J. Biol. Chem. 281, 6874-6883 (2006).

20. Tam, S. Y. et al. RabGEF1 is a negative regulator of mast cell activation and skin inflammation. Nat. Immunol. 5, 844-852 (2004).

21. Marichal, T. et al. Guanine nucleotide exchange factor RABGEF1 regulates keratinocyte-intrinsic signaling to maintain skin homeostasis. J. Clin. Invest. 126, 4497-4515 (2016).

22. Kuhn, R., Lohler, J., Rennick, D., Rajewsky, K. \& Muller, W. Interleukin-10-deficient mice develop chronic enterocolitis. Cell 75, 263-274 (1993).

23. Barnett, M. P. et al. Changes in colon gene expression associated with increased colon inflammation in interleukin-10 gene-deficient mice inoculated with Enterococcus species. BMC Immunol. 11, 39 (2010).

24. Hansen, J. J., Holt, L. \& Sartor, R. B. Gene expression patterns in experimental colitis in IL-10 deficient mice. Inflamm. Bowel Dis. 15, 890 (2009).

25. Russ, A. E. et al. Gene expression changes in the colon epithelium are similar to those of intact colon during late inflammation in interleukin-10 gene deficient mice. PLOS ONE 8, e63251 (2013).

26. Keubler, L. M., Buettner, M., Hager, C. \& Bleich, A. A Multihit Model: colitis lessons from the interleukin-10-deficient mouse. Inflamm. Bowel Dis. 21, 1967-1975 (2015).

27. Paul, G., Khare, V. \& Gasche, C. Inflamed gut mucosa: downstream of interleukin10. Eur. J. Clin. Invest. 42, 95-109 (2012).

28. Yan, Y. et al. Temporal and spatial analysis of clinical and molecular parameters in dextran sodium sulfate induced colitis. PLoS One 4, e6073 (2009).

29. Kim, E. et al. MouseNetv2: a database of gene networks for studying the laboratory mouse and eight other model vertebrates. Nucleic Acids Res. 44, D848-D854 (2016).

30. Schwerk, J. et al. Generation of mouse small intestinal epithelial cell lines that allow the analysis of specific innate immune functions. PloS ONE 8, e72700 (2013).

31. Kondo, T., Kawai, T. \& Akira, S. Dissecting negative regulation of Toll-like receptor signaling. Trends Immunol. 33, 449-458 (2012).

32. O'Neill, L. A. \& Bowie, A. G. The family of five: TIR-domain-containing adaptors in Toll-like receptor signalling. Nat. Rev. Immunol. 7, 353-364 (2007).

33. Kamada, N., Seo, S. U., Chen, G. Y. \& Nunez, G. Role of the gut microbiota in immunity and inflammatory disease. Nat. Rev. Immunol. 13, 321-335 (2013).
34. Dethlefsen, L., McFall-Ngai, M. \& Relman, D. A. An ecological and evolutionary perspective on human-microbe mutualism and disease. Nature 449, 811-818 (2007).

35. Uhlig, H. H. et al. Differential activity of IL-12 and IL-23 in mucosal and systemic innate immune pathology. Immunity 25, 309-318 (2006).

36. Nunes, N. S. et al. Temporal clinical, proteomic, histological and cellular immune responses of dextran sulfate sodium-induced acute colitis. World J. Gastroenterol. 24, 4341-4355 (2018).

37. Eichele, D. D. \& Kharbanda, K. K. Dextran sodium sulfate colitis murine model: An indispensable tool for advancing our understanding of inflammatory bowel diseases pathogenesis. World J. Gastroenterol. 23, 6016-6029 (2017).

38. Lotz, M. et al. Postnatal acquisition of endotoxin tolerance in intestinal epithelial cells. J. Exp. Med. 203, 973-984 (2006).

39. Otte, J. M., Cario, E. \& Podolsky, D. K. Mechanisms of cross hyporesponsiveness to Toll-like receptor bacterial ligands in intestinal epithelial cells. Gastroenterol 126, 1054-1070 (2004).

40. Rhee, S. H. et al. Pathophysiological role of Toll-like receptor 5 engagement by bacterial flagellin in colonic inflammation. Proc. Natl Acad. Sci. USA 102, 13610-13615 (2005).

41. Alkim, C., Alkim, H., Koksal, A. R., Boga, S. \& Sen, I. Angiogenesis in inflammatory bowel disease. Int J. Inflam. 2015, 970890 (2015).

42. Danese, S. \& Gasbarrini, A. Chemokines in inflammatory bowel disease. J. Clin. Pathol. 58, 1025-1027 (2005).

43. Wang, D., Dubois, R. N. \& Richmond, A. The role of chemokines in intestinal inflammation and cancer. Curr. Opin. Pharm. 9, 688-696 (2009).

44. Shea-Donohue, T. et al. Mice deficient in the CXCR2 ligand, CXCL1 (KC/GRO alpha), exhibit increased susceptibility to dextran sodium sulfate (DSS)-induced colitis. Innate Immun. 14, 117-124 (2008).

45. Parhar, K., Ray, A., Steinbrecher, U., Nelson, C. \& Salh, B. The p38 mitogenactivated protein kinase regulates interleukin-1 beta-induced IL- 8 expression via an effect on the IL-8 promoter in intestinal epithelial cells. Immunology $\mathbf{1 0 8}$ 502-512 (2003)

46. Waterhouse, C. C., Joseph, R. R., Winsor, G. L., Lacombe, T. A. \& Stadnyk, A. W. Monocyte chemoattractant protein-1 production by intestinal epithelial cells in vitro: a role for p38 in epithelial chemokine expression. J. Interferon Cytokine Res. 21, 223-230 (2001)

47. Kawai, T. \& Akira, S. The role of pattern-recognition receptors in innate immunity: update on Toll-like receptors. Nat. Immunol. 11, 373-384 (2010).

48. Gay, N. J., Symmons, M. F., Gangloff, M. \& Bryant, C. E. Assembly and localization of Toll-like receptor signalling complexes. Nat. Rev. Immunol. 14, 546-558 (2014).

49. Kalesnikoff, J. et al. RabGEF1 regulates stem cell factor/c-Kit-mediated signaling events and biological responses in mast cells. Proc. Natl Acad. Sci. USA 103 2659-2664 (2006).

50. Montero-Melendez, T., Llor, X., Garcia-Planella, E., Perretti, M. \& Suarez, A. Identification of novel predictor classifiers for inflammatory bowel disease by gene expression profiling. PloS ONE 8, e76235 (2013).

51. Madison, B. B. et al. Cis elements of the villin gene control expression in restricted domains of the vertical (crypt) and horizontal (duodenum, cecum) axes of the intestine. J. Biol. Chem. 277, 33275-33283 (2002).

52. Dieleman, L. A. et al. Chronic experimental colitis induced by dextran sulphate sodium (DSS) is characterized by Th1 and Th2 cytokines. Clin. Exp. Immunol. 114, 385-391 (1998).

53. Erben, U. et al. A guide to histomorphological evaluation of intestinal inflammation in mouse models. Int J. Clin. Exp. Pathol. 7, 4557-4576 (2014). 